This item was submitted to Loughborough's Research Repository by the author.

Items in Figshare are protected by copyright, with all rights reserved, unless otherwise indicated.

\title{
High resolution simulations of high Reynolds number jets with microjet injection
}

PLEASE CITE THE PUBLISHED VERSION

http://dx.doi.org/10.2514/6.2013-2263

\section{PUBLISHER}

Published by the American Institute of Aeronautics and Astronautics, Inc. @ the author(s)

\section{VERSION}

AM (Accepted Manuscript)

\section{PUBLISHER STATEMENT}

This work is made available according to the conditions of the Creative Commons Attribution-NonCommercialNoDerivatives 4.0 International (CC BY-NC-ND 4.0) licence. Full details of this licence are available at: https://creativecommons.org/licenses/by-nc-nd/4.0/

\section{LICENCE}

CC BY-NC-ND 4.0

\section{REPOSITORY RECORD}

Rife, M.E., and Gary J. Page. 2016. "High Resolution Simulations of High Reynolds Number Jets with Microjet Injection”. figshare. https://hdl.handle.net/2134/22504. 


\title{
High Resolution Simulations of High Reynolds Number Jets with Microjet Injection
}

\author{
M. E. Rife* and G. J. Page ${ }^{\dagger}$ \\ Department of Aeronautical \& Automotive Engineering, Loughborough University, Leicestershire, UK
}

\begin{abstract}
Large eddy simulations have been performed of a Mach 0.9 jet at a Reynolds number of 1.3 million for both a clean and microjet injected configurations. Two numerical grids were used for the simulations differing in the number of azimuthal cells. The first with 720 cells and an azimuthal clustering near the microjet injection locations, and the other with 1440 cells and a uniform azimuthal cell spacing that matches the finest cell for the clustered case. The grids contained 100 million and 200 million cells respectively. A standard Smagorinsky sub-grid scale model was used together with a synthetic trip in the nozzle shear layer. The non-uniform grid with 720 cells azimuthally showed a variation in laminar to turbulent transition location that was a function of the clustering, with later transition in the coarser regions. However, this had little detrimental impact on mean velocity distributions further downstream. The results of the simulations were compared with PIV experimental data and good agreement of mean radial velocity and turbulent kinetic energy profiles were obtained. The microjets caused a deformation of the shear layer, reducing the radial location of peak turbulence kinetic energy in-line with the microjets. Additionally, the shear layer is translated away from the jet centreline between the microjets and becomes flat in the regions between the microjets. A Ffowcs Williams-Hawking technique was used to propagate the unsteady pressure fluctuations to the far-field. Spectral data at downstream and sideline observer locations indicated the presence of a high-frequency peak in the microjet case which is consistent with the size of the microjets. The microjets provide a blockage effect to the main jet and the peak is probably a shedding like behaviour. Overprediction of overall sound pressure levels by 6-8 dB was found when compared to the experimental data, however, the correct behaviour with observer angle was captured and more importantly the microjets showed a reduction in OASPL of around 2 dB across a range of angles, similar to the experiment results.
\end{abstract}

\section{Nomenclature}

$\begin{array}{llll}c_{o} & \text { Speed of sound } & \vec{u} & \text { Fluid velocity } \\ D_{j} & \text { Jet Nozzle diameter } & \vec{U}_{j} & \text { Mean jet velocity } \\ D_{m j} & \text { Microjet diameter } & \vec{v} & \text { Surface velocity } \\ \text { LBM } & \text { Lattice-Boltzmann Methodology } & \vec{x} & \text { Observer location } \\ \text { LES } & \text { Large Eddy Simulation } & \vec{y} & \text { Source location } \\ M & \text { Mach number } & \delta_{i j} & \text { Kronecker delta } \\ M_{0} & \text { Free stream Mach number } & \boldsymbol{\eta} & \text { FWH surface } \\ \dot{m} & \text { Mass flow rate } & \rho & \text { Density } \\ \text { MILES } & \text { Monotonically Integrated Large Eddy } & \rho_{0} & \text { Ambient density } \\ & \text { Simulation } & \sigma_{i j} & \text { Reynolds stress } \\ p_{i j} & \text { Compressive stress tensor } & & \\ \text { PIV } & \text { Particle Image Velocimetry } & & \\ R_{D_{j}} & \text { Reynolds number based on jet diameter } & & \\ T_{i j} & \text { Lighthill stress tensor } & & \end{array}$

\footnotetext{
* Reasearch Student

${ }^{\dagger}$ Senior Lecturer, AIAA Member
} 


\section{Introduction}

Strict international noise regulations, coupled with pressure from communities near airports and local governments, continue to drive the requirement for quieter aircraft. During take-off, engine noise is the dominant source in the overall aircraft noise with jet noise being the majority contributor to engine noise. As a result, a significant amount of work has been performed with the goal of reducing jet noise. Two common methods investigated for achieving a noise reduction are the use of chevrons or microjets. Chevrons are a modification to the trailing edge of the jet nozzle, adding serrations to the edge of the nozzle. Microjets are small high pressure jets located around the nozzle edge that introduce high momentum fluid into the shear layer of the otherwise round jet. Both of these devices have been shown experimentally to introduce streamwise vorticity into the shear layer and can result in a reduction of far field noise. ${ }^{1-4}$ Microjets have an advantage over static devices such as chevrons in that they can be disabled when noise reduction is not necessary, such as during cruise, thus eliminating fuel burn penalties during the majority of the mission.

The first large eddy simulation of a jet with microjets was conducted by Åberg et al. ${ }^{5}$ in 2007 . This work was performed on a 1 million element unstructured mesh. The main jet had an exit Mach number of 0.5 and a Reynolds number of $6 \times 10^{5}$. 16 microjets were included in the microjet simulation with an area ratio of $1.5 \%$ and a momentum ratio of $1.8 \%$. Noise prediction was achieved by solving the acoustic source terms of the inhomogeneous wave equation and linearly interpolating onto an acoustic mesh that extended to the far-field. Flow-field results showed a small extension of the potential core length and a downstream translation and reduction in size of peak root mean square velocity $\left(u_{r m s}^{\prime}\right)$ values in the early shear layer. Acoustic results were compared to experimental results, though the source and parameters of the experiment were not specified. Nevertheless, sideline OASPL was predicted to be increased by $0.1 \mathrm{~dB}$ whereas the experimental values compared to showed up to a $0.3 \mathrm{~dB}$ reduction in sideline noise. Downstream noise at $30^{\circ}$ from the jet axis was predicted to be reduced by $0.2 \mathrm{~dB}$. This agrees well with the experimental value for the lower pressure microjets, though less well for higher pressure microjets, which showed a $0.4 \mathrm{~dB}$ reduction. It should be noted that the injected to main jet momentum ratio is very low (1.8\%), compared to the more common values employed by Alkislar et al. ${ }^{2}$ of 2.5 (i.e. $250 \%$ ). Despite the very low momentum of the microjets, a small impact on the flow-field and sound-field was observed.

Huet et al. ${ }^{6}$ performed a numerical study of continuous and pulsed microjets on hot and cold jets. Using the Monotonically Integrated Large Eddy Simulation (MILES) approach, they simulated a cold Mach 0.9 jet with a Reynolds number of $1.0 \times 10^{6}$ and a Mach 0.64 hot jet with a Reynolds number of $3.2 \times 10^{5}$. Both jets were simulated with 12 evenly spaced microjets inclined by $45^{\circ}$ to the jet axis. The mass flow rates of the continuous microjets was $4.8 \times 10^{-4} \mathrm{~kg} / \mathrm{s}$ in both cases. The simulations were performed on a hybrid mesh containing $4.5 \times 10^{6}$ cells. The microjets were included into the simulation by modifying the source terms of the mass, momentum and energy equations to include the mass injection and convection of the microjets. This was applied to one hexahedral cell that acted as the source of each microjet. Noise prediction was computed using a FWH surface method The far-field noise of the clean jets was overpredicted by around 6 $\mathrm{dB}$ compared to experimental values. The overprediction of the far-field noise may be attributable to the underprediction of the potential core length by $30 \%$ coupled with an over-estimation of turbulent kinetic energy. This is likely due to an initially laminar shear layer originating at the nozzle exit. The addition of the microjets showed a reduction of sideline noise by approximately $1.5 \mathrm{~dB}$ while little change was seen in the downstream direction for the cold jet and a $2 \mathrm{~dB}$ increase for the hot jet downstream noise. This contrasts with the general trend found experimentally, showing microjets to cause greater reduction to downstream observer angle far-field noise and little to negative benefit at sideline stations.

Lew, Najafiyazdi, and Mongeau ${ }^{7}$ used a Lattice-Boltzmann Methodology LES (LBM-LES) to simulate a Mach 0.5 jet with a Reynolds number of $1 \times 10^{5}$ with 18 microjets. LBM-LES is different from traditional LES as it does not solve the Navier-Stokes equations but rather solves particle distribution functions. The simulation was performed on a cartesian mesh. Grid refinement in the region of the microjets allowed for 20 cells across the face of the microjets. Flow-field results compared well with experimental data, the potential core length matched well with experiments of a similar nozzle and operating condition. Activating the microjets was shown to provide an increase in the potential core length of about 1 jet diameter. Centreline $u_{r m s}^{\prime}$ also matched well to experimental work and the peak value was shown to be reduced by approximately $6 \%$ with the application of microjets. The use of LBM-LES and the cartesian mesh used in this work generates some limitations. LBM-LES is limited to low Mach number flows (approximately Mach 0.5 or less), though Lew et al. do provide evidence of successful simulations up to Mach 0.9. Additionally, the cartesian mesh created some artefacts in the solution. Small regions of low velocity flow are visible near the nozzle exit 
in regions where a cell wall is parallel to the nozzle wall (i.e. along the coordinate axes). Furthermore, the velocity contours of the clean jet have a distinct non-circular shape. Despite these artefacts, acoustic results obtained from a Ffowcs Williams-Hawking surface method showed close agreement with experimental results. The clean jet noise was overpredicted by $1 \mathrm{~dB}$ for observer angles less than $80^{\circ}$ and introducing the microjets to the simulation provided a $1.5 \mathrm{~dB}$ reduction in far-field noise.

Najafi-Yazdi et $a l^{8}$ conducted a large eddy simulation of a Mach 0.9 unheated jet with a Reynolds number of $4 \times 10^{5}$ fitted with eight microjets inclined by $60^{\circ}$ to the jet axis. The microjet parameters were similar to the experimental work of Alkislar et $a l^{2}$ The simulation was performed on a curvilinear mesh and did not include the nozzle geometry. Far-field noise results overpredicted the baseline overall sound pressure level (OASPL) by $6 \mathrm{~dB}$. Inclusion of the microjets showed around a $4 \mathrm{~dB}$ reduction in OASPL compared to the $0.5-1 \mathrm{~dB}$ reduction reported by Alkislar et al. ${ }^{2}$ The accuracy of the results is hindered by the choice to exclude the nozzle from the simulation domain. Najafi-Yazdi et al. note that the potential core length is reduced when the microjets are active. Although the potential core length is not available from the experimental work, other works show an increase in potential core length caused by the presence of microjets. Furthermore, an increase in potential core length agrees with a reduction in shear layer turbulent kinetic energy. The initial shear layer in the work is clearly shown to be laminar for a couple of jet diameters before undergoing transition to a fully turbulent state. This is a common problem with simulations that exclude the nozzle geometry and is exacerbated by the disparity between the experimental jet Reynolds number and that employed in the simulation. Finally, the effect of the microjets on the shear layer shape reported by the simulation does not match the experimental results. Alkislar et al. report that each microjet penetrates through the shear layer causing a counter-rotating vortex pair to exist on the high-speed side of the shear layer for each microjet. Contrasting with this, at $x=1 D_{j}$ Najafi-Yazdi et al. show only 4 counter rotating vortex pairs which exist on the outside of the jet shear layer.

Enomoto et al. ${ }^{9}$ perfromed a series of simulations of a Mach 0.9 jet with a Reynolds number of $1 \times 10^{6}$ fitted with 18 microjets. They performed two simulations with the microjets active, changing the microjet mass flow ratio from $3.36 \times 10^{-4}$ to $8.86 \times 10^{-4}$. Their simulations were aimed to match the experimental work of Castelain et al. ${ }^{4}$ The simulation was performed on a mesh containing 476 million grid points. Included in the simulation was a $1 D_{j}$ length of a simple straight pipe nozzle fitted with a numerical boundary layer trip to aid in a realistic transition to a fully turbulent shear layer. Microjets were implemented as $4 \times 4$ inlets and were inclined $45^{\circ}$ to the jet axis. The acoustic results of the clean jet simulation were within $5 \mathrm{~dB}$ of the experimental results. Results with the microjets active were not compared to experimental work, though a 2 $\mathrm{dB}$ reduction at low frequencies was found at an observer angle $30^{\circ}$. Overall sound pressure levels were found to be minimally reduced at downstream observer locations for the high mass flow ratio case and unaffected in the lower mass flow ratio simulation. Sideline overall sound pressure levels were found to increase with the microjets active, with more penalty arising in the higher mass flow ratio case.

Shur et al. ${ }^{10}$ conducted a series of simulations of a Mach 0.9 jet with and without microjets in static and flight conditions at Mach 0.2, typical of take-off and landing. This is the first investigation of the effect of forward flight conditions on microjets known to the authors. The simulations were conducted on a structured multiblock grid containing 7.6 million cells with $515 \times 101 \times 144$ cells in the axial, radial, and azimuthal directions, respectively. The results of the simulations showed accurate flow-field results with the microjets activated in the static condition. With the microjets active, the large-scale turbulent structures are seen to be suppressed; likewise, the turbulent kinetic energy in the shear layer was noticeably reduced when the microjets were activated. Similarly, the far-field noise was shown to be reduced by approximately 1-2 dB for all observer locations. However, the effect of the microjets on the flow and sound-field in flight condition was remarkably disheartening. The work showed that the reduction of TKE in the shear layer was negligible. As suggested by this, the far-field noise results were effectively indistinguishable between the microjet and clean jet case. Analysis of the broadband noise spectra at both a downstream and sideline observer location showed little effect on the low frequency components while the location of the cross-over frequency remained unchanged compared to the static conditions simulation. This indicates that the highfrequency penalty of the microjets will remain without any associated low frequency benefit. Finally, the authors noted the existence of two counter-rotating vortex pairs generated by each microjet, opposed to only one pair commonly reported by both experiments and simulations. The work indicates a high intensity but rapidly dissipated vortex pair that originates near the microjets and exists principally on the high-speed side of the shear layer. A second, lower intensity but longer lasting, vortex pair was found to be generated slightly downstream of the microjet injection and existed closer to the half-velocity line. The existence of 
two vortex pairs is suggested to account for the complex, non-monotonic nature of the vortex pairs reported by Alkislar et al. ${ }^{2}$

Recently, work was undertaken by Rife and Page ${ }^{11}$ on a 15 million element cylindrical mesh originally created for clean jet simulations. The work included the introduction of microjets into the simulation and investigated the effect of these microjets on flow-field characteristics and two-point spatio-temporal correlations. In this work the microjets were rather poorly resolved, being applied as $2 \times 2$ pressure inlets. The present work builds on the work of Rife and Page, with two sets of simulations having been carried out, using $100-200$ million element meshes. These simulations are based on the experimental work of Alkislar et $a l .,{ }^{2}$ maintaining both main jet and microjet geometry and operating conditions. Flow-field and acoustic far-field results will be presented, investigating the effects of the microjets, with comparison to experimental data.

This paper will begin with a review of the computational methodology is presented in Section II, starting with a review of the numerical solver in Section II.A, followed by a summary of the Ffowcs Williams-Hawking method of noise prediction in Section II.B. Simulation properties are presented in Section III including details of the numerical grids in Section III.A. The results will be provided in Section IV, split into aerodynamic results in Section IV.A and aeroacoustic results provided in Section IV.B. Finally, conclusions will follow in Section V.

\section{Computational Methodology}

The computational methodology of the work will be presented in this section. This will cover a brief overview of the solver details, followed by a discussion of the two computational grids used in the work and will close with a review of the Ffowcs Williams-Hawking method of noise prediction employed for this work.

\section{A. Numerical Solver}

Solution of the filtered Navier-Stokes equations is obtained through a finite volume pressure-based method on a multi-block grid. A finite volume method is employed as it guarantees conservation of fluxes through the control volume. The solver uses primary variables defined as quantities per unit volume, not per unit mass. As a result, momentum becomes a primary variable which the conservation equation is applied to and velocity is then calculated from momentum and density. When used on pressure-based algorithms this method benefits the accuracy for compressible flows. It is typical to use density-based approaches for high-speed flows. However, these methods become inefficient and unstable at low Mach numbers. The pressure-based approach is well suited for the present work as simulations of jets contain regions of very high speed and very low speed flows. Pressure correction is employed using the SIMPLE method and Rhie-Chow smoothing is used to avoid pressure-velocity decoupling. For LES, the standard Smagorinsky model is used for the sub-grid scale stress tensor.

Spatial discretization is performed using a high-order upwind scheme that is similar to the QUICK scheme with an explicit sub-grid scale model. Upwind based schemes have been used for jet noise simulations by several researchers in the past. Andersson et al. ${ }^{12-15}$ used a third-order upwind scheme in their works, with good fluidic and acoustic results. Karabasov et al. ${ }^{16}$ employed a second order upwind numerical scheme to perform a large eddy simulation. The results of the simulation were used to inform an acoustic analogy for far-field noise prediction. Very accurate spectral results were obtained from the LES and acoustic analogy. Shur et al. ${ }^{17,18}$ used an upwind biased numerical scheme. In these works, Shur et al. compared the effect of numerical schemes on the development of an unforced jet with a Reynolds number of $10^{4}$. A third-order upwind scheme with the subgrid model disabled produced an initial shear layer that was laminar for almost 4 jet diameters, while with a fifth-order upwind scheme (again with subgrid model disabled) produced an earlier transition to a fully turbulent shear layer, indicating a reduction in numerical dissipation and better resolution of the turbulence. It is well known that upwind schemes are dissipative in nature, and the MILES approach used by Shur et al. clearly demonstrates the increasing effects on small scale turbulence suppression with lower order upwind schemes. However, central difference schemes are notoriously dispersive, causing significant unrealistic fluctuations to occur in the regions of sharp gradients and discontinuities (e.g. shock waves). When accurate far-field noise prediction is a goal of the simulation the dispersive nature of a central difference scheme can easily contaminate the results. As such, the more dissipative, though significantly less dispersive, upwind scheme available in Delta was used for all simulations. 
Temporal advancement is achieved through the use of an implicit backward Euler method. Near-wall length-scale damping is added using a modified van Driest damping treatment.

\section{B. Far-Field Noise Prediction}

Formulation 1A of the FWH equation, as presented by Brentner and Farassat, ${ }^{19}$ was used for far-field noise prediction. Although their method allows for the motion of a moving surface, such as one around a rotating blade, some simplifications were possible as the surfaces used surrounding the jets in the present work are stationary. Formulation $1 \mathrm{~A}$ is most simply written as the summation

$$
p^{\prime}(\vec{x}, t)=p_{T}^{\prime}(\vec{x}, t)+p_{L}^{\prime}(\vec{x}, t)+p_{Q}^{\prime}(\vec{x}, t)
$$

The terms on the right hand side of Equation 1 are referred to as the thickness, loading, and quadrupole noise, respectively. The thickness noise is a result of monopole sources while the loading noise is caused by dipole sources. The quadrupole term, $p_{Q}^{\prime}$, requires solving a volume integral over the region outside of the surface. The suitability of neglecting the quadrupole term was discussed by Morgans. ${ }^{20}$ In order for the quadrupole term to be sufficiently small to be able to be neglected, Lighthill's stress tensor, $T_{i j}$, must be small outside of the FWH surface and the observer must be in the acoustic far-field. In the case of a free jet in a stationary medium it is easy to ensure that $T_{i j}$ remains small outside the surface by controlling the location of the surface. As the fluctuating parts of the terms defining $T_{i j}$ are those that will contribute to any acoustic noise generation, if the surface is located such that any significant fluctuating flow features are contained within the surface then the value of $T_{i j}$ outside the surface will remain small, and the quadrupole term can be neglected in the noise prediction.

The values of $p_{T}^{\prime}$ and $p_{L}^{\prime}$ at an observer location, $\vec{x}$, are obtained from Equations 2 and 3 and are summations of integrals over the FWH surface at retarded time.

$$
\begin{aligned}
4 \pi p_{T}^{\prime}(\vec{x}, t)=\int_{f=0} & {\left[\frac{\rho_{0}\left(\dot{U}_{n}+U_{\dot{n}}\right)}{r\left(1-M_{r}\right)^{2}}\right]_{r e t} d S+\int_{f=0}\left[\frac{\rho_{0} U_{n}\left(r \dot{M}_{r}+c\left(M_{r}-M^{2}\right)\right)}{r^{2}\left(1-M_{r}\right)^{3}}\right]_{r e t} d S } \\
4 \pi p_{L}^{\prime}(\vec{x}, t) & =\frac{1}{c} \int_{f=0}\left[\frac{\dot{L}_{r}}{r\left(1-M_{r}\right)^{2}}\right]_{r e t} d S+\int_{f=0}\left[\frac{L_{r}-L_{M}}{r^{2}\left(1-M_{r}\right)^{2}}\right]_{r e t} d S \\
& +\frac{1}{c} \int_{f=0}\left[\frac{L_{r}\left(r \dot{M}_{r}+c\left(M_{r}-M^{2}\right)\right)}{r^{2}\left(1-M_{r}\right)^{3}}\right]_{r e t} d S
\end{aligned}
$$

In Equations 2 and 3 the term $M$ is the modulus of the Mach number vector of the source. In the case of the FWH surface, the source is considered to be each cell on the surface. In the present work the surface is stationary, therefore the Mach number of the source is zero, that is $M=0$. As a result, the radiated component of $M, M_{r}$, and its first time derivative, $\dot{M}_{r}$, are both zero. Furthermore, the term $L_{M}$ is defined as $L_{M}=L_{i j} M_{i}$ and thus is zero in the present work. Finally, the term $U_{\dot{n}}$ is defined as $U_{\dot{n}}=U_{i} \dot{\hat{n}}$ where $\hat{n}_{i}$ is the $i$ component of the unit normal vector to the surface. Again, as the surface is stationary there is no variation to the unit normal vector with time, thus $\dot{\hat{n}}$ and $U_{\dot{n}}$ both become zero for all instances. These zero values allow for Equations 2 and 3 to be significantly reduced. The results of the simplifications for the case where the Ffowcs Williams-Hawking surface is fixed in space are given below.

$$
\begin{gathered}
4 \pi p_{T}^{\prime}(\vec{x}, t)=\int_{f=0}\left[\frac{\rho_{0} \dot{U}_{n}}{r}\right]_{r e t} d S \\
4 \pi p_{L}^{\prime}(\vec{x}, t)=\frac{1}{c} \int_{f=0}\left[\frac{\dot{L}_{r}}{r}\right]_{r e t} d S+\int_{f=0}\left[\frac{L_{r}}{r^{2}}\right]_{r e t} d S
\end{gathered}
$$

Equations 4 and 5 are the basic foundation for the Ffowcs Williams-Hawking surface method used in the present work. In these equations, $\rho_{o}$ is the ambient density of the undisturbed fluid, and $c$ is the ambient 


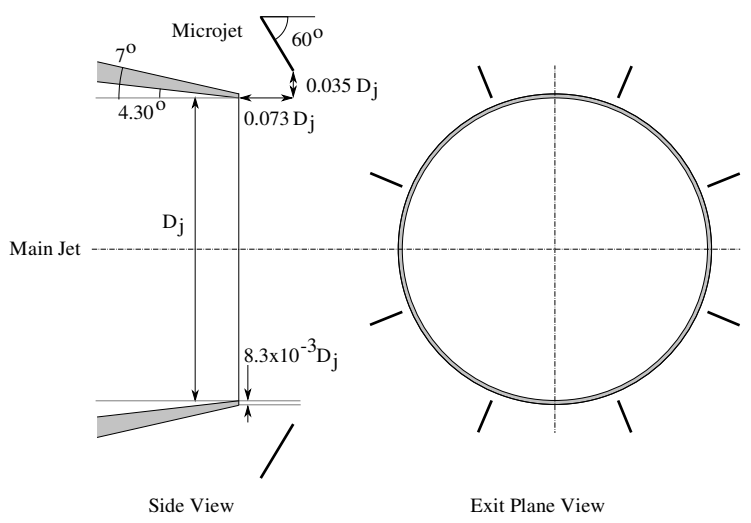

Figure 1. Diagram showing nozzle and microjet geometry

speed of sound. The term $r$ is the distance between the observer and the source, $r=|\vec{x}-\vec{y}|$, where $\vec{x}$ is the observer location and $\vec{y}$ is the source location.

The terms $U$ and $L$ are a modified velocity vector and modified stress tensor, respectively. They are defined as:

$$
\begin{gathered}
U_{i}=\left[1-\left(\rho / \rho_{o}\right)\right]+\left(\rho u_{i} / \rho_{o}\right) \\
L_{i j}=P_{i j} \hat{n}_{j}+\rho u_{i} u_{j} \hat{n}_{j}
\end{gathered}
$$

In Equations 4 and $5, \dot{U}_{n}=\dot{U}_{i} \hat{n}_{i}$ where the over dot indicates a time derivative. Similarly, $L_{r}=L_{i j} \hat{r}_{i}$ and $\dot{L}_{r}=\dot{L}_{i j} \hat{r}_{i}$. Finally, $\rho$ is the instantaneous density of the source, and $P_{i j}$ is the compressive stress tensor, defined as $P_{i j}=\left(p-p_{o}\right) \delta_{i j}$ where $p$ is the instantaneous pressure at the source, $p_{o}$ is the ambient pressure and $\delta_{i j}$ is the Kronecker delta.

Equations 2, 3, and their simplified forms, 4 and 5, all have the subscript ret after the bounding square brackets. This indicates that the integral is to be performed at retarded time. If an observer is located in an acoustic field that is the result of multiple sources at different locations then sound waves from each different source need to leave at different times in order to reach the observer at the same time. This source dependent emission time is known as retarded time, and is often denoted as $\tau=t-\frac{r}{c}$.

\section{Simulation Parameters}

Simulations of a jet fitted with eight equally spaced microjets have been performed. The simulations are closely based on the experimental work of Alkislar et al. ${ }^{2}$ In all simulations, the nozzle has a diameter of $69.85 \mathrm{~mm}$ and a lip thickness of $0.58 \mathrm{~mm}$. The main jet has an exit Mach number of 0.9 and the Reynolds number is $1.3 \times 10^{6}$, based on exit velocity and nozzle diameter. The eight uniformly distributed microjets are located $5 \mathrm{~mm}$ downstream of the nozzle exit and $2.5 \mathrm{~mm}$ radially from the internal edge of the nozzle lip. A diagram of the nozzle geometry and microjet location is shown in Figure 1. The microjets have an internal diameter of $800 \mu \mathrm{m}$ and are inclined to the jet axis by 60 degrees. In the experiment, the microjets had a fully expanded Mach number of 1.5, giving a nozzle pressure ratio of 3.67. This resulted in an microjet to main jet momentum flux ratio of 2.5. Care was taken when creating the mesh to ensure the internal convergence angle, diameter, and lip thickness of the experimental nozzle were maintained. Likewise, boundary conditions were set to ensure the simulated jet parameters matched the experimental values.

\section{A. Computational Grid}

Two sets of simulations were performed on two cylindrical grids, both designed to match the nozzle geometry used in the experimental work of Alkislar et al. ${ }^{2}$ Both grids have the same geometrical size, extending $3.75 D_{j}$ upstream of the nozzle exit, $30 D_{j}$ downstream of the nozzle exit, and $10 D_{j}$ in the radial direction. One grid contains $707 \times 203 \times 720$ (axial $\times$ radial $\times$ azimuthal) elements with bunching in the azimuthal direction. 


\begin{tabular}{|l|c|c|c|}
\hline Case ID & Microjets Active & Azimuthal Cell Count & Grid Cell Count \\
\hline AKB720 & $\boldsymbol{x}$ & 720 & 100 million \\
\hline MJ_AKB720 & $\boldsymbol{J}$ & 720 & 100 million \\
\hline AKB1440 & $\boldsymbol{X}$ & 1440 & 200 million \\
\hline MJ_AKB1440 & $\boldsymbol{J}$ & 1440 & 200 million \\
\hline
\end{tabular}

Table 1. Simulation case IDs and descriptions

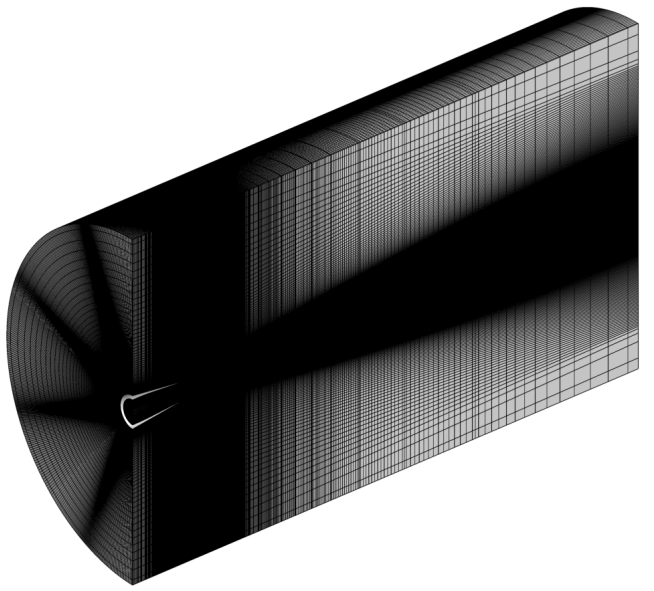

(a) Half of the AKB1440 mesh

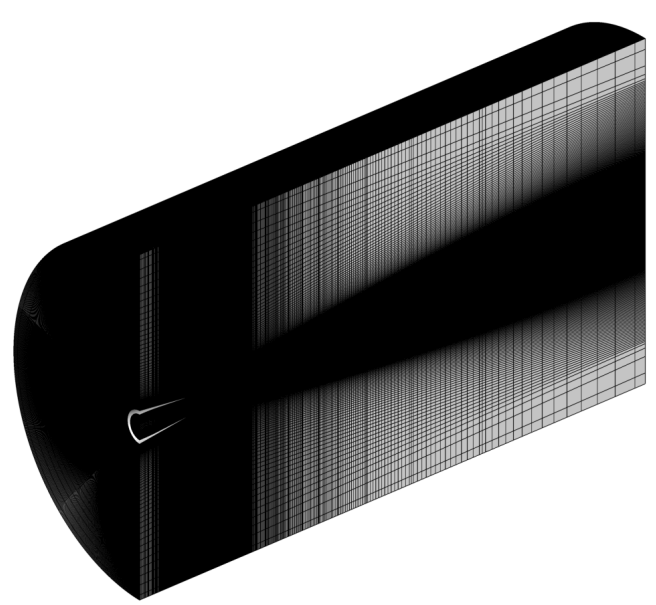

(b) Half of the AKB1440 mesh

Figure 2. Half views of AKB720 and AKB1440 grids

This azimuthal bunching was implemented to allow high resolution in the regions of the microjets while trying to minimise computational costs. The angular size of each element in the azimuthal direction varies sinusoidally from $0.25^{\circ}$ to $0.75^{\circ}$ with the finer elements located in-line with the microjet locations. The finest cell size in the radial direction is located at the nozzle lip and is $0.4 \times 10^{-3} D_{j}$. Likewise, the finest cell size in the axial direction is located in line with the nozzle exit and has a length of $1.5 \times 10^{-3} D_{j}$.

The second, increased resolution grid, doubles the number of azimuthal elements while removing the azimuthal bunching. The resulting grid contains 1,440 uniformly distributed elements azimuthally giving an angular cell width of $0.25^{\circ}$ for all cells. The domain dimensions remain the same as the first grid. Consequently the grids contain 100 million and 200 million elements, respectively both having the same multi block topology of 47 blocks. To aid with discussion, the 100 million element grid with azimuthal bunching will be referred to as AKB720 and the 200 million element grid case will be referred to as AKB1440 from here on. When the microjets are active the cases will be referred to as MJ_AKB720 and MJ_AKB1440. This naming convention is outlined in Table 1.

The two grids are shown in Figure 2 by slicing the domain in half so as to allow the clustering at the nozzle and in the shear layer to be visible. For the AKB720 grid (Figure 2(a)) the clustering in the azimuthal direction is clearly seen and this is further highlighted by a cross-section at the nozzle exit shown in Figure 3(a). It should be noted that in Figure 3(b), the dark quatrefoil in the centre of the mesh is an artifact of the plotting anti-aliasing routines and the mesh is actually uniform in the azimuthal direction. The dark ring in both figures coincides with the nozzle lip, where the cells with the shortest radial length exist.

Figure 4 provides a close-up view of the mesh near the nozzle lip. 20 cells exist across the nozzle lip thickness of $8.3 \times 10^{-3} D_{j}$, or $0.58 \mathrm{~mm}$, with a constant radial spacing of $0.4 \times 10^{-3} D_{j}$, matching the wall spacing inside the nozzle. The fine radial grid spacing is important to resolve the high gradients and small turbulent structures of the wall boundary layer inside the nozzle and the free shear layer downstream; 


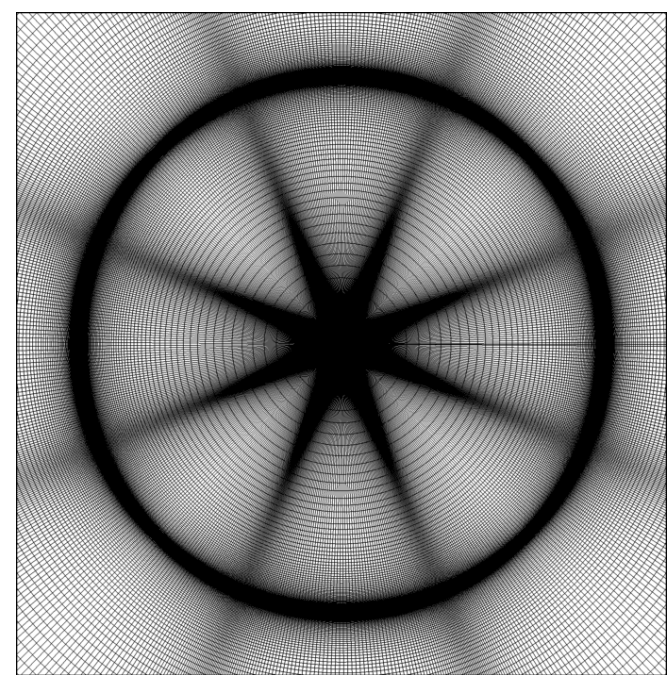

(a) 100 million element mesh

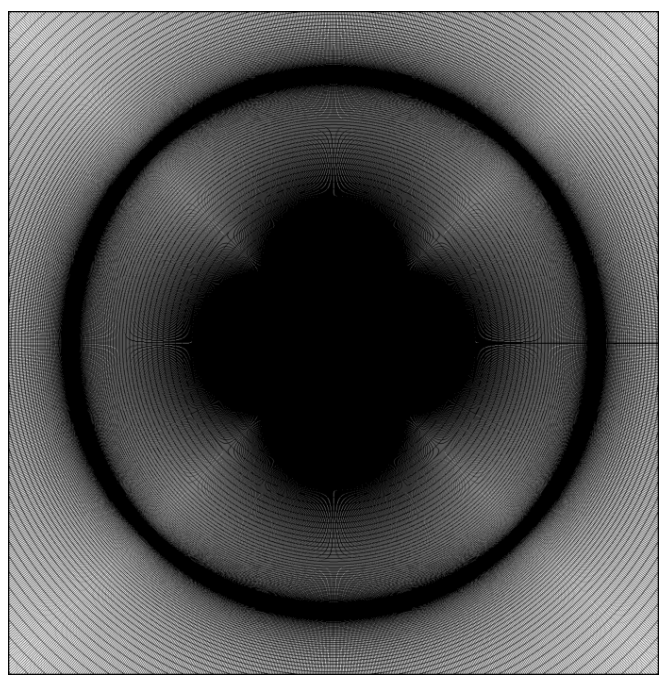

(b) 200 million element mesh

Figure 3. Cross section of computational grids showing azimuthal clustering

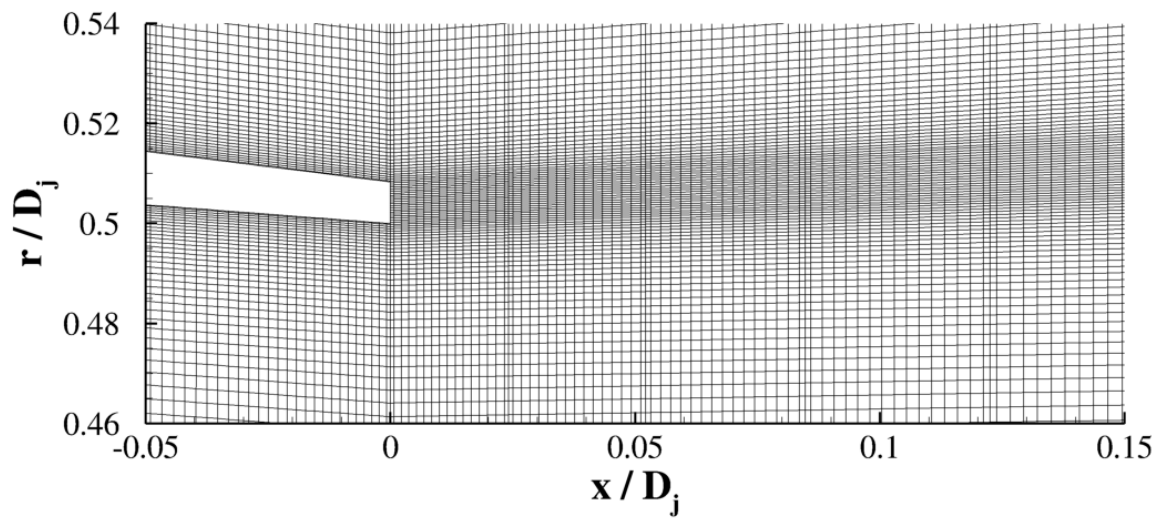

Figure 4. Close-up view of mesh near nozzle exit

however, as the shear layer grows in size the spacing is increased downstream to reflect the reduction in mean gradient and the increase in size of the dominant turbulent eddies. The $0.25^{\circ}$ angular spacing results in a linear azimuthal dimension of $0.2 \times 10^{-3} D_{j}$ at the nozzle lip.

\section{Results}

\section{A. Aerodynamic results}

Instantaneous iso-surfaces of Mach 0.25 from the AKB720 and AKB1440 simulations are shown in Figures 5 and 6 , respectively. Both simulations include a numerical boundary layer 'trip' within the nozzle located half of a diameter upstream of the nozzle exit. The trip is used to introduce weakly correlated turbulence near the wall which persists at a low level at the nozzle exit plane. Although this turbulence is weak, it causes a realistic generation of the initial shear layer and avoids the creation of unrealistic axisymmetric structures near the nozzle exit.

Figure 5(a) shows the region near the nozzle exit for the AKB720 simulation, with a closer view provided in Figure 5(b). It is clear from the figures that an early and natural transition to a fully turbulent shear layer is captured in this simulation. Closer inspection of Figure 5(b) reveals that the axial location of the 


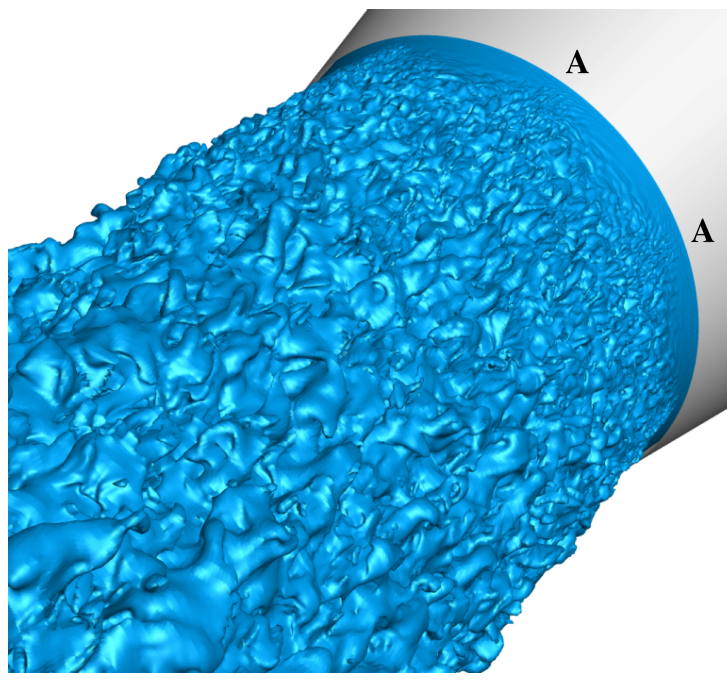

(a) Overview of early shear layer development

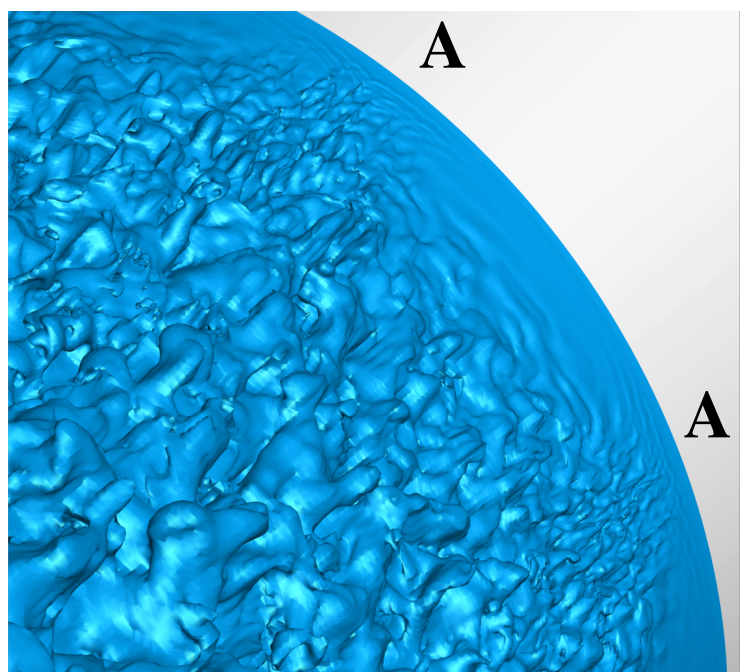

(b) Closeup showing azimuthal variation in shear layer transition location

Figure 5. Instantaneous iso-surfaces of Mach 0.25 (AKB720)

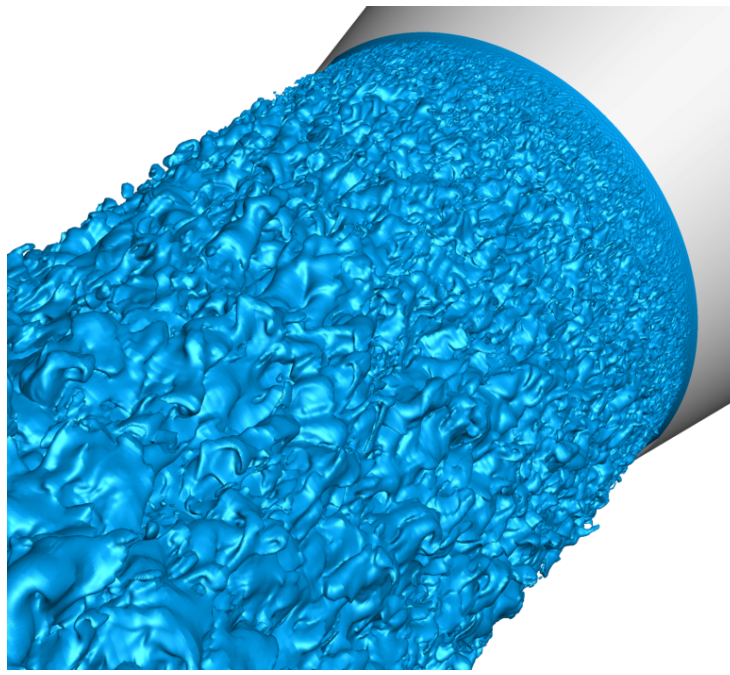

(a) Initial shear layer

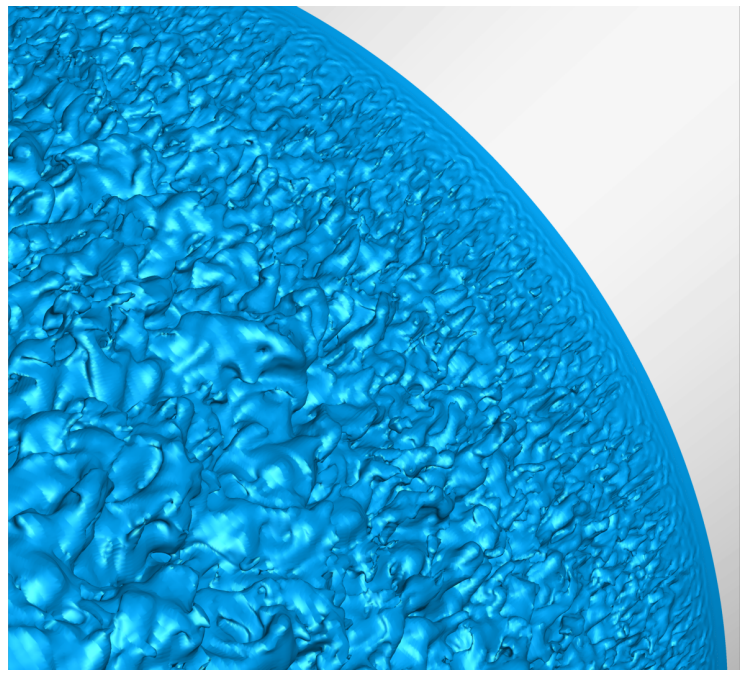

(b) Closeup showing azimuthal uniformity in shear layer

Figure 6. Instantaneous iso-surfaces of Mach 0.25 (AKB1440)

transition to a turbulent shear layer varies with azimuthal location. An earlier transition to turbulence occurs in regions where the azimuthal bunching has reduced the cell width (indicated by 'A' in Figure 5), while the more delayed transition occurs in areas with larger cells. As expected, a smaller cell size allows for the resolution of finer scale turbulent structures. It is believed that the azimuthal component of the early turbulent structures plays an important role in the transition to a fully turbulent shear layer. It is also possible that the reduction in length scale in the Smagorinsky sub grid scale model (which is based on the cube root of cell volume) also plays a role as this will locally reduce sub-grid scale viscosity. Examining Figures 6(a) and (b) it is seen that this anomalous variation in transition location is not present in the uniform azimuthal spacing AKB1440 case.

The effect of the azimuthal cell size on flow variables can also be seen from the instantaneous vorticity magnitude within the shear layer near the nozzle exit, as shown in Figure 7. The location of the AKB720 slice is aligned with the largest azimuthal cells and the shear layer clearly remains laminar for longer than the AKB1440 case before large-scale coherent structures develop and propagate downstream. For the AKB1440 


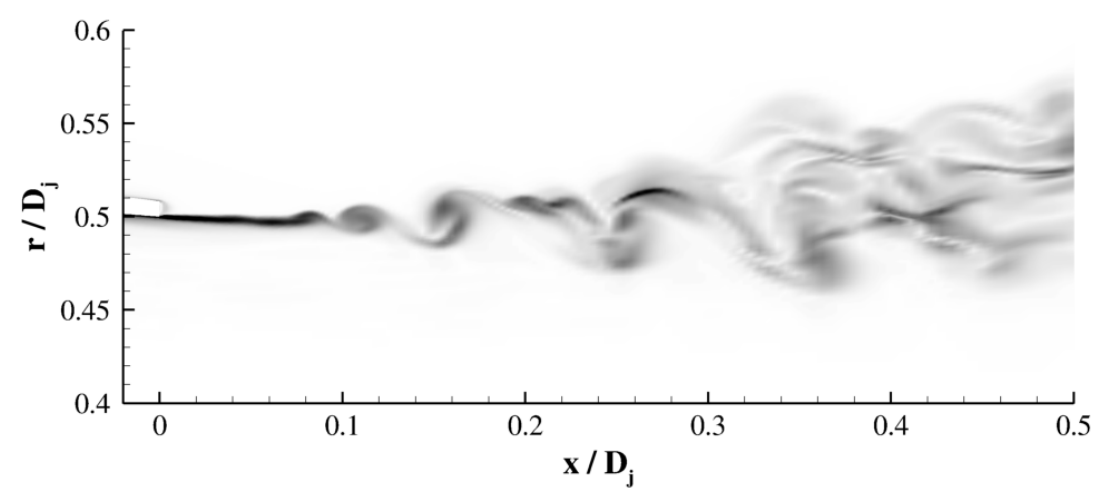

(a) AKB720

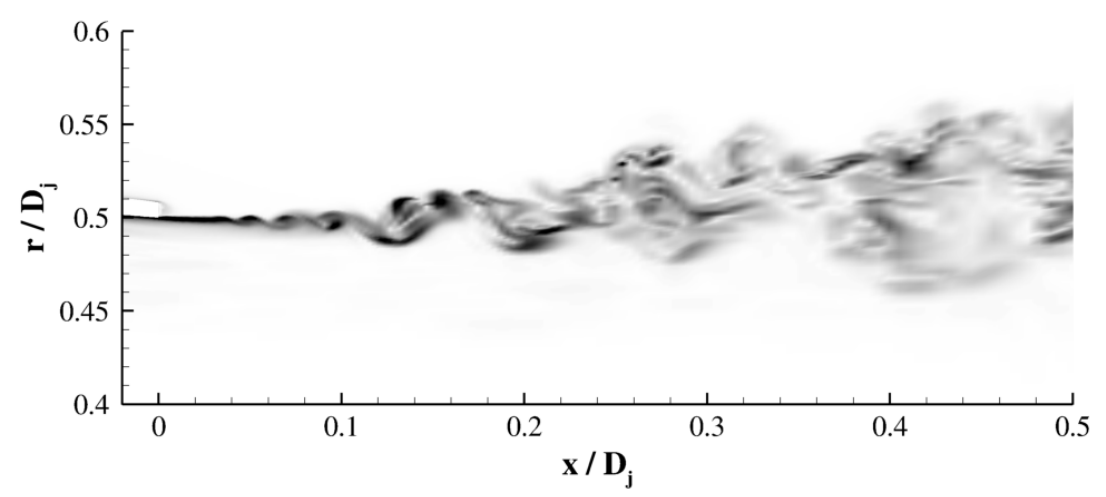

(b) AKB1440

Figure 7. Instantaneous contours of vorticity magnitude near the nozzle exit

case, there is a transition to a fully turbulent shear layer from $0.05-0.1 D_{j}$ with no dominant large-scale structures. This indicates that the azimuthal size of the cells is an important factor to resolve structures in the early shear layer development.

Figure 8 shows instantaneous contours of vorticity magnitude across a microjet. Compared to Figure 7(a), it is evident that the introduction of the microjet causes a significant increase to the thickness of the shear layer downstream of the injection location. The shear layer from the nozzle lip impinges on the microjet creating an unsteady interaction and a feedback mechanism that creates vortical structures immediately downstream of the nozzle lip in the region $0-0.05 D_{j}$. Downstream the microjet blockage has a wake like behaviour with significantly larger turbulent structures than are visible from non-microjet visualisations.

Figure 9 shows the mean iso-surface of Mach 0.25 for the MJ_AKB1440 simulation. The microjets are shown to penetrate through the iso-surface, disturbing the circular nature of the jet. It appears from Figure 9 that the microjets pierce the shear layer and exist on the high-speed side of the shear layer. However, it is difficult to confirm this from the figure. Lateral slices of Mach number allow for a more thorough investigation of the effects of the microjets on the velocity distribution through the jet.

Contours of mean Mach number are shown for three early axial stations for MJ_AKB720 and MJ_AKB1440 simulations in Figures 10 and 11, respectively. Figures 10(a) and 11(a) show mean Mach number of AKB1440 and MJ_AKB1440 at $x / D_{j}=0.1$. This is just downstream of the location where the microjets first interact with the shear layer. It can be seen that the microjets cause some outward deflection of the shear layer near the microjet injection points at this early location. At $x / D_{j}=0.25$ it is seen in Figures 10(b) and 11(b) that the microjets penetrate through the shear layer and cause perturbations to exist on the high speed side 


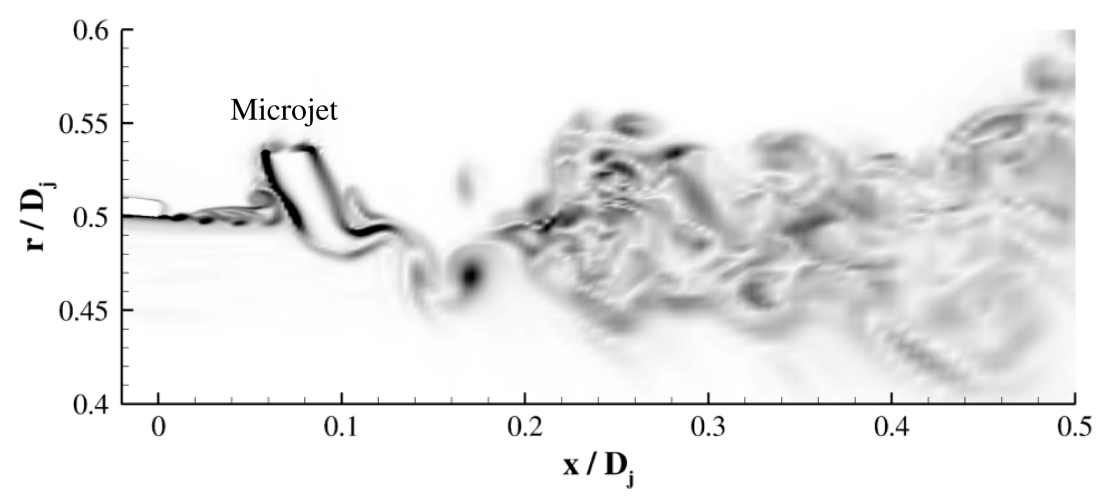

(a) MJ_AKB720

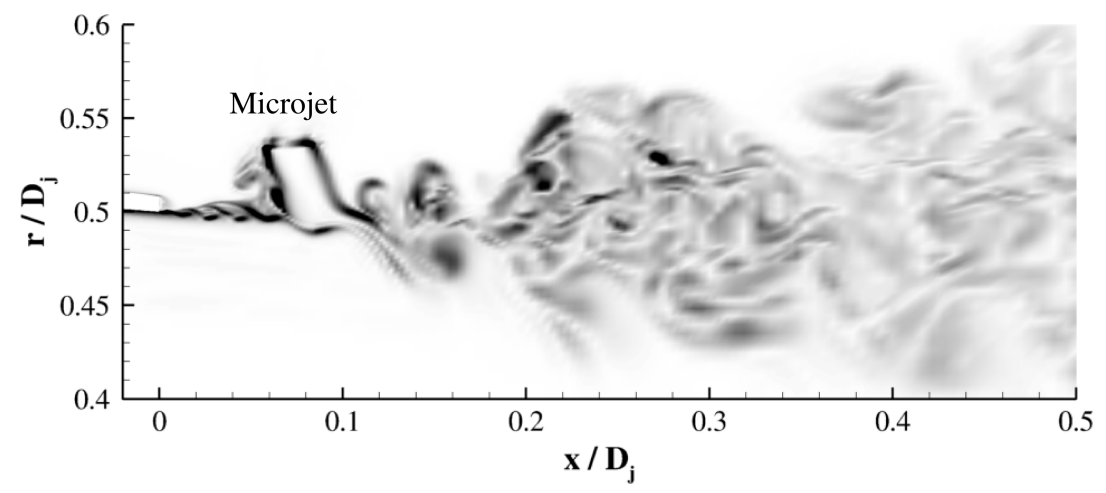

(b) MJ_AKB1440

Figure 8. Contours of vorticity magnitude with microjets active

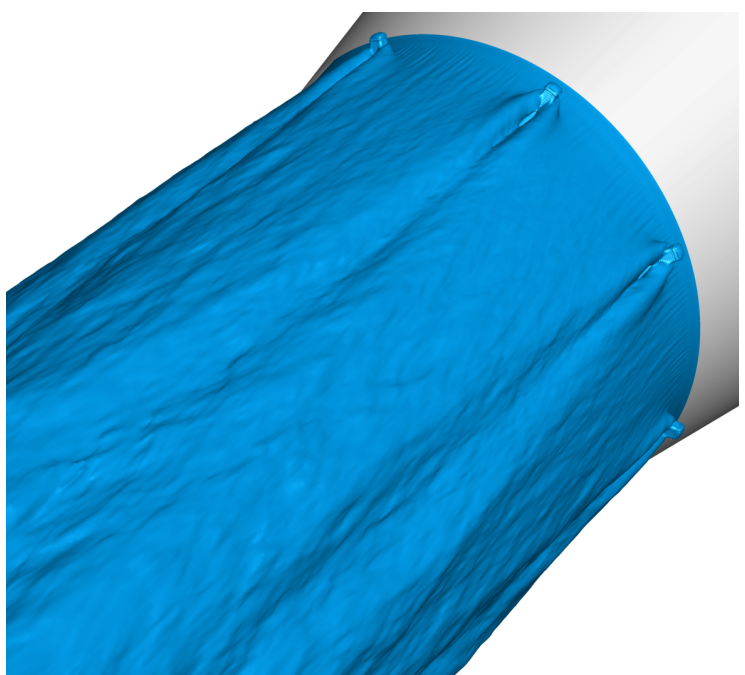

Figure 9. Mean iso-surface of Mach 0.25 MJ_AKB1440 


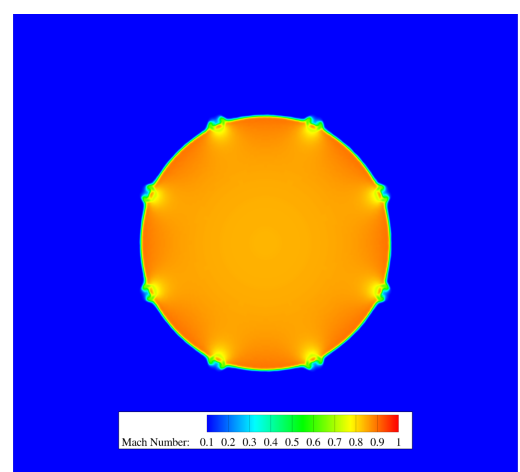

(a) $x / D_{j}=0.1$

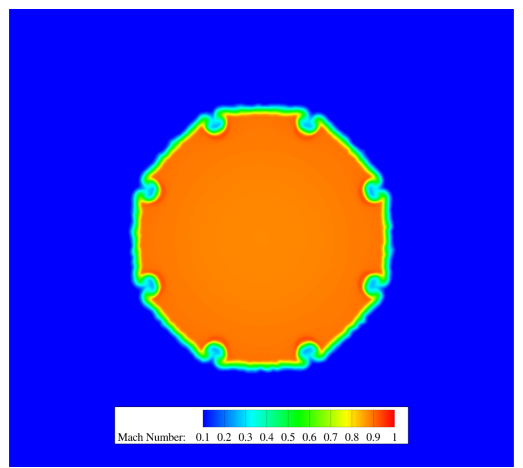

(b) $x / D_{j}=0.25$

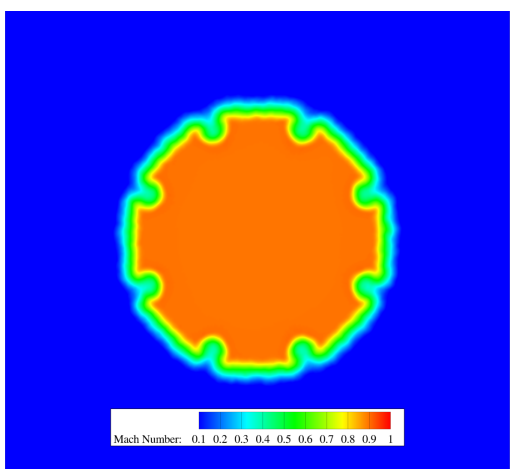

(c) $x / D_{j}=0.5$

Figure 10. Contours of mean Mach number for MJ_AKB720.

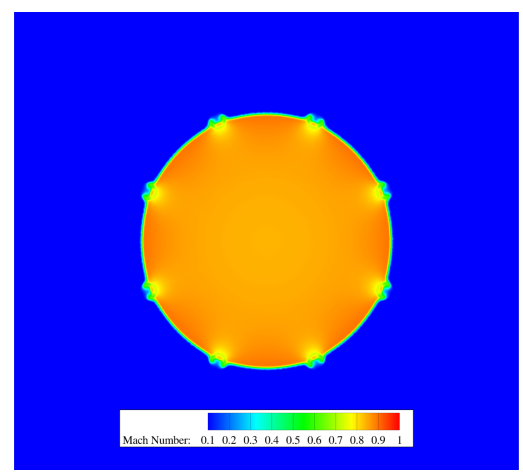

(a) $x / D_{j}=0.1$

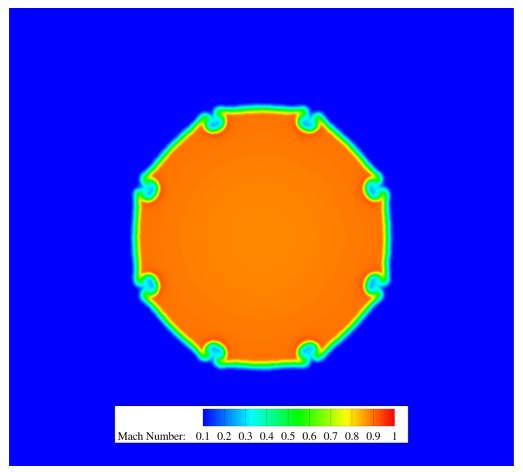

(b) $x / D_{j}=0.25$

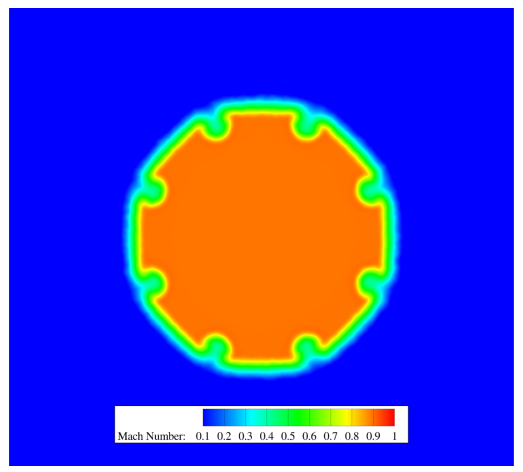

(c) $x / D_{j}=0.5$

Figure 11. Contours of mean Mach number for MJ_AKB1440.

of the shear layer. Furthermore, the circular shape of the shear layer is altered in between the microjets and a more linear shear layer appears to exist in this region in both simulations. Looking at Figure 10(c) and 11(c) it is clear that the effect of the microjets continues to exist on the high speed side of the shear layer and the regions between the microjets continues to form a clear octagonal shape. Overall, the visible difference between the contours of the two simulations are indiscernible.

Clean jet radial velocity profiles are shown in Figure 12(a) to (c). The experimental velocity profiles obtained by Alkislar et al. are provided in the figures along with the results of the AKB720 and AKB1440 simulations. The profiles from AKB720 were obtained from the region of the finest cells, though the difference between profiles obtained from different azimuthal locations is negligible. At $x=1 D_{j}$ the velocity decay rate through the shear layer is slightly underpredicted by both simulations. This may be attributable to the small co-flow that was included in the simulation for stability purposes. This co-flow will reduce the difference between the potential core and the ambient air, thereby potentially reducing the velocity decay rate through the shear layer. Despite these issues, the velocity profiles at this station agree well with the experimental profile.

At $x=2 D_{j}$, (Figure 12(b)), and $x=5 D_{j}$ (Figure 12(c)) the two simulations yield very similar results and compare well to the experimental data. Earlier discrepancies in velocity decay rates are minimised and the profiles coincide well with each other.

Figure 13 provides velocity profiles at the same axial locations for the experimental microjet results and the results of the MJ_AKB1440 simulation. Profiles are given both in-line with the microjet injection location and between two microjets. The clean AKB1440 profiles are included as a baseline reference.

Figure 13(a) shows the velocity profiles at $x=1 D_{j}$. With the microjets active, the velocity profile between the microjet injection location is shown to be radially displaced from the baseline in both the experiment and the simulation. In-line with the microjets a reduction in the radial extent of the jet core was simulated which closely matches the experimental values. However, the extent of the velocity reduction 


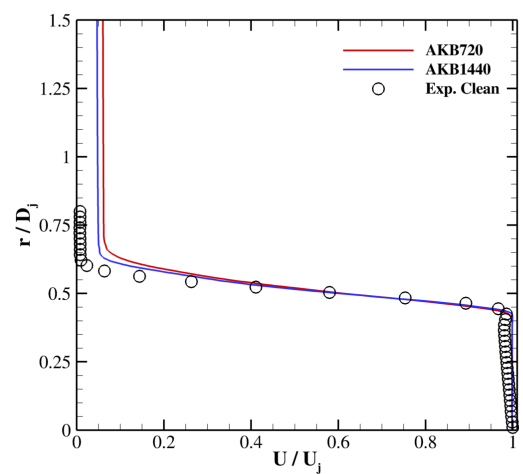

(a) $x=1 D_{j}$

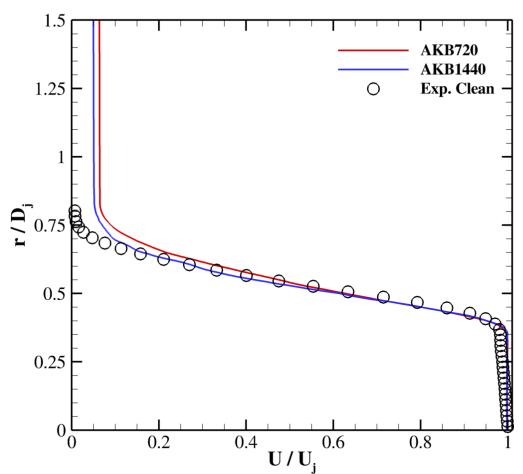

(b) $x=2 D_{j}$

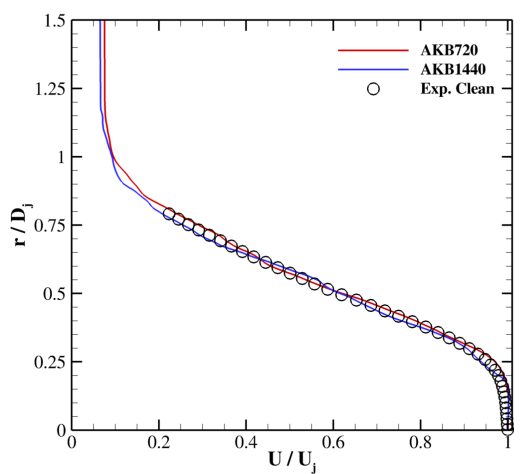

(c) $x=5 D_{j}$

Figure 12. Radial velocity profiles for clean jet experimental and simulation results.

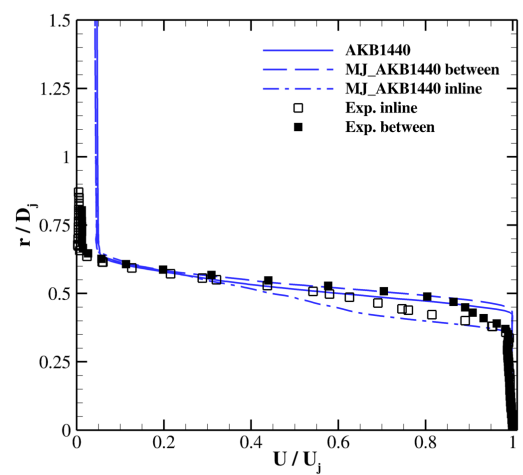

(a) $x=1 D_{j}$

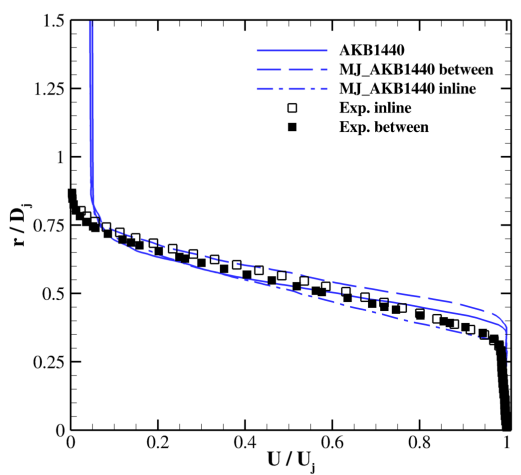

(b) $x=2 D_{j}$

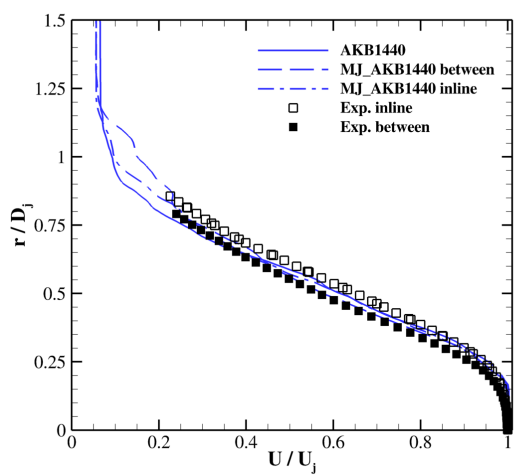

(c) $x=5 D_{j}$

Figure 13. Radial velocity profiles for microjet experimental and simulation results.

through the microjet wake is larger in the simulation as compared to the experimental values.

At $x=2 D_{j}$, (Figure 13(b)), the profile between the microjets is shifted away from the jet centreline in the simulation. In-line with the microjet injection points a wake, due to the effective blockage from the microjet penetration, reduces the radial dimension of the jet core. The simulations overpredict the extent of this wake, taking longer for the velocity to match the baseline profile. In the experimental case the velocity profile in-line with the microjets crosses the baseline profile near $0.5 r / D_{j}$, with the microjet profile maintaining a higher velocity in the outer regions of the shear layer than the baseline. This is not captured in the simulations, where the microjet velocity profile only manages to match the basline profile at the outer most regions of the shear layer.

Figure 13(c) shows the radial velocity profiles at $x=5 D_{j}$. At this location the effect of the microjets on the velocity profiles in the simulation has diminished and the profiles collapse onto each other. The experimental profiles, however, do not collapse and rather the profile in-line with the microjets extends further in the radial direction than the profile between the microjets. This is an unexpected result and is likely due to a small shift in the jet centreline in the experiment due to the test facilities as discussed by Alkislar et al. ${ }^{2}$

Overall, the radial profiles of the simulations agree well with the experiment. Furthermore, there is little variation between the AKB720 and AKB1440 clean jet velocity profiles. The profiles of the MJ_AKB720 and MJ_AKB1440 simulations also closely match and thus the MJ_AKB720 profiles were not shown for the sake of clarity.

Figures 14 to 17 show contours of turbulent kinetic energy at $x / D_{j}=0.1,0.25$, and 0.5 for the four simulations. Figure 14 shows the turbulent kinetic energy contours near the nozzle exit for the AKB720 simulation. At $x / D_{j}=0.25$ regions of heightened TKE are found in the areas with the larger cell spacing. The intensity of the increased TKE appears to be dissipated by $x / D_{j}=0.5$, though the contours appear to 


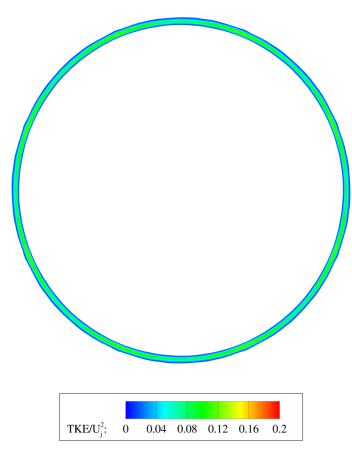

(a) $\operatorname{AKB} 720 x / D_{j}=0.1$

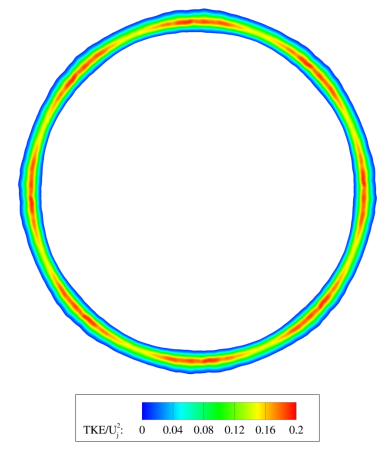

(b) $\operatorname{AKB720x/D_{j}=0.25}$

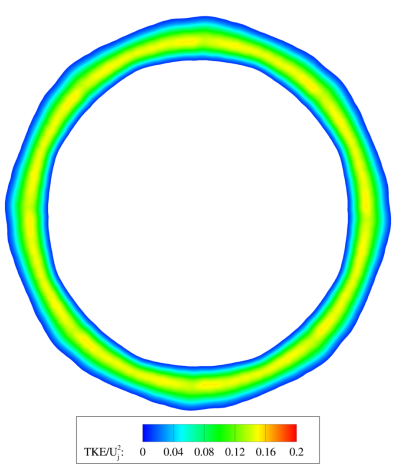

(c) $\operatorname{AKB} 720 x / D_{j}=0.5$

Figure 14. Contours of turbulent kinetic energy for AKB720 simulation

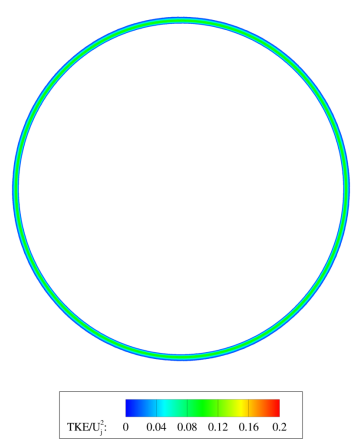

(a) $\operatorname{AKB} 1440 x / D_{j}=0.1$

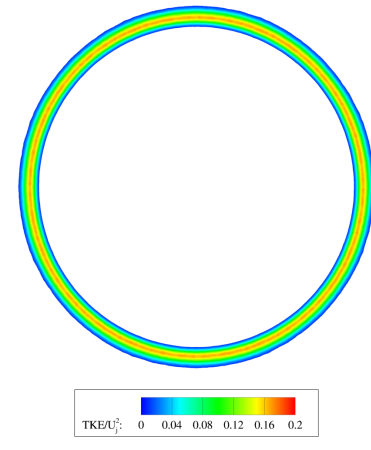

(b) $\operatorname{AKB} 1440 x / D_{j}=0.25$

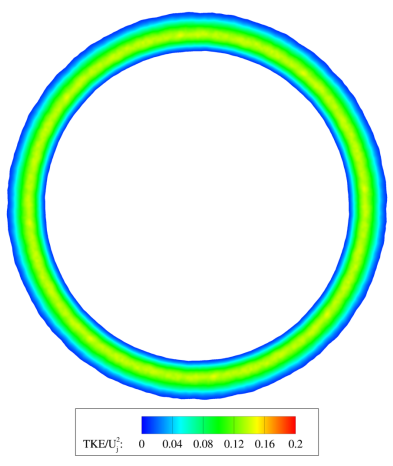

(c) $\operatorname{AKB} 1440 x / D_{j}=0.5$

Figure 15. Contours of turbulent kinetic energy for AKB1440 simulation

have a slight non-circular shape. The TKE contours for the AKB1440 simulation are provided in Figure 15. In the AKB1440 simulation the value of TKE does not vary significantly with azimuthal angle around the centreline. The anomalous peaks in turbulent kinetic energy can be attributed to the larger turbulent scales in the region of lower resolution in the AKB720 simulation. Referring back to Figure 7, it can be seen that the vortical structures visible at $x / D_{j}=0.25$ are larger in the AKB720 simulation than in the AKB1440 simulation. These larger scales will have more kinetic energy than the smaller scales in the regions of the AKB720 mesh with the highest resolution, or in the AKB1440 simulation.

Figure 16 and Figure 17 illustrate the turbulent kinetic energy contours at the same early axial locations for the two simulations. Little difference is observed at $x / D_{j}=0.1$, although by $x / D_{j}=0.25$ the level of TKE between the microjets is clearly larger for MJ_AKB720 than MJ_AKB1440. This increased value of TKE in the MJ_AKB720 simulation is maintained through to $x / D_{j}=0.5$. This is the same mechanism that produced anomalous peaks in TKE at the locations of largest cells for the clean case

Radial turbulent kinetic energy profiles at $x=1,2$ and $5 D_{j}$ for the clean jet experiment and AKB720 and AKB1440 simulations are given in Figures 18(a) to (c), respectively, with the profiles from the AKB720 simulation obtained from the region of highest resolution.

At $x=1 D_{j}$ the simulations show a peak turbulent kinetic energy level of slightly more than $0.015 U_{j}^{2}$ while the experimental peak is closer to $0.006 U_{j}^{2}$. The reason for this large discrepancy is unclear, though it may be that the experimental PIV resolution is not sufficiently fine in the near nozzle region to fully resolve the stresses in this region. The PIV data comes from planes divided into $120 \times 80$ cells, with as few as 4 cells across the shear layer. Another cause of the different levels could be the numerical trip applied in the simulations. As mentioned earlier, this trip helps the shear layer to transition to a turbulent state early on in the development. It is possible that the experiment, without any boundary layer trip inside the nozzle, had a later transition to a fully turbulent shear layer, thus reducing the turbulent kinetic energy compared to the turbulent shear layer in the simulations. 


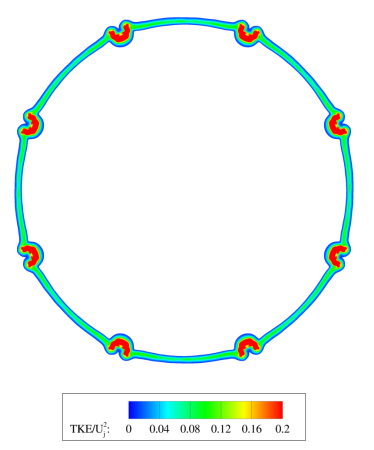

(a) MJ_AKB720 $x / D_{j}=0.1$

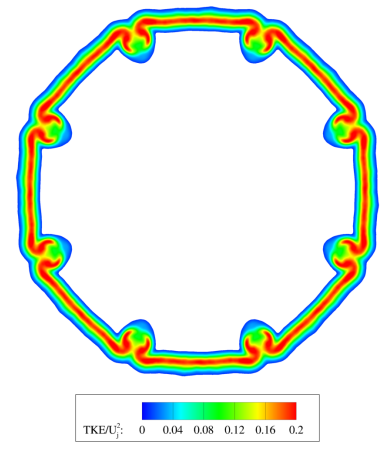

(b) MJ_AKB720 $x / D_{j}=0.25$

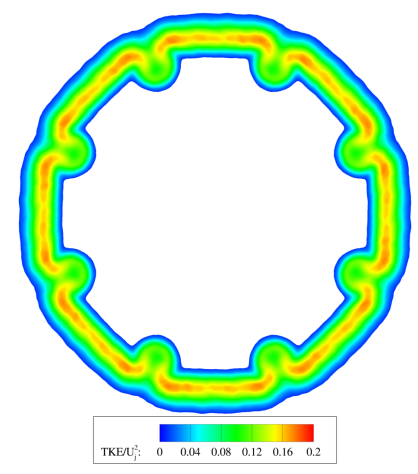

(c) MJ_AKB720 $x / D_{j}=0.5$

Figure 16. Contours of turbulent kinetic energy for MJ_AKB720 simulation

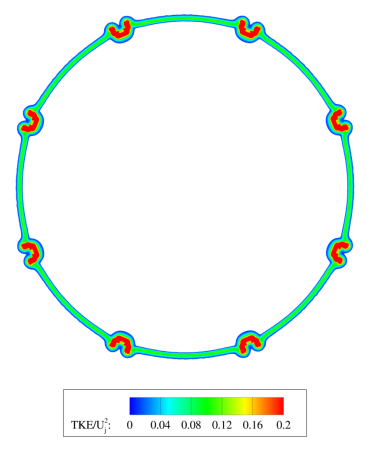

(a) MJ_AKB1440 $x / D_{j}=0.1$

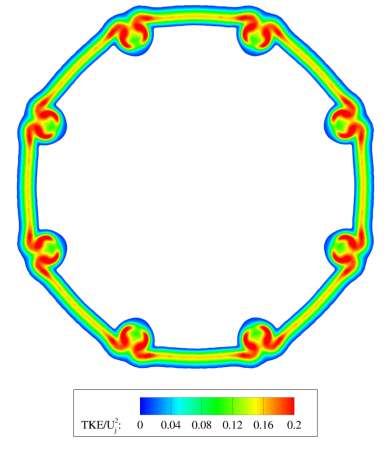

(b) MJ_AKB1440 $x / D_{j}=0.25$

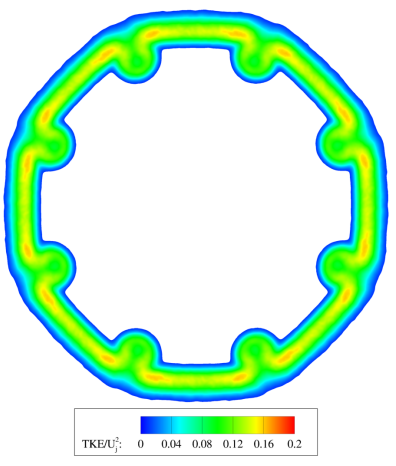

(c) MJ_AKB1440 $x / D_{j}=0.5$

Figure 17. Contours of turbulent kinetic energy for MJ_AKB1440 simulation

At $x=2 D_{j}$ the profiles of turbulent kinetic energy from both simulations compare very well to the experimental profile with both the shape and amplitude of the profiles matching well.

A

Figures 19(a) to (c) show the experimental and MJ_AKB1440 turbulent kinetic energy profiles at $x=$ 1,2 and $5 D_{j}$. As with the radial velocity profiles, the radial TKE profiles of both simulations are in good agreement. As such, only the AKB1440 profiles are shown as a baseline.

At $x=1 D_{j}$ the experimental profiles show little difference between the peak amplitude of TKE. However, the radial location of the peak is positioned further from the centreline when between the microjets. Compared to the experimental baseline, the peak TKE has also been increased for both profiles by introducing the microjets. The simulation profile between the microjets shows an increase in peak TKE and a radial shift away from the centreline. When in-line with the microjets the peak TKE is reduced compared to the baseline and is moved toward the jet centreline.

At $x=2 D_{j}$ the experimental profile between the microjets shows a larger peak amplitude than in-line with the microjets and exhibits a radial outward shift. Between the microjets the experimental peak amplitude is of comparable value to the clean experimental peak value. The simulation captures similar results, with the profile between the microjets exhibiting a peak at a radial location further from the centreline than the clean simulation peak, but with a similar value. Between the microjets, the maximum TKE is less than the AKB1440 baseline value and exists close to the radial location of the baseline. Compared to the experiment, the profile between the microjets overpredicts the peak TKE, while in-line with the microjets the peak TKE is underpredicted.

As with the experimental velocity profiles at $x=5 D_{j}$, the profile in-line with the microjets shows a peak further from the jet centreline than the profile between the microjets. The causes for this were discussed above. The effect of the microjets is to reduce the peak turbulent kinetic energy from the experimental baseline at this location. The simulation shows little difference between the AKB1440 baseline and the two 


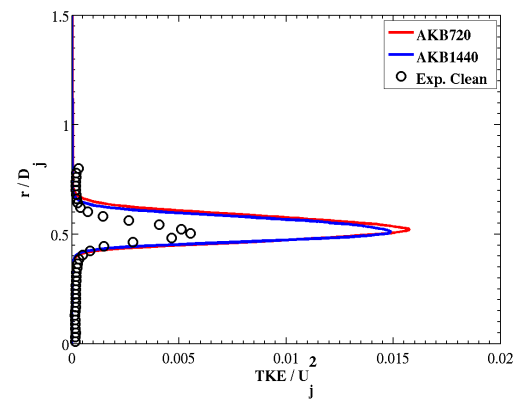

(a) $x=1 D_{j}$

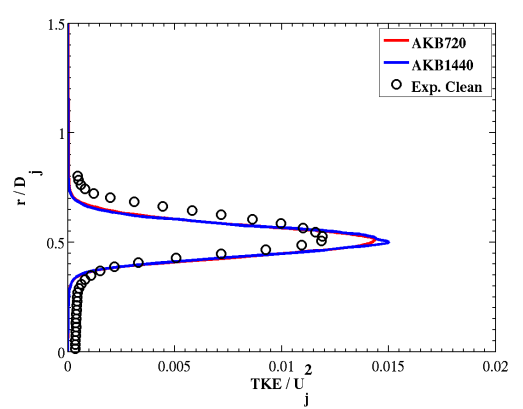

(b) $x=2 D_{j}$

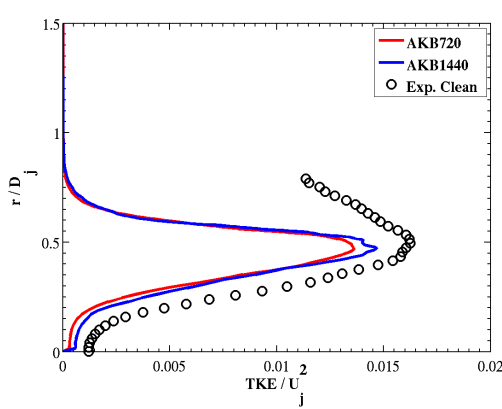

(c) $x=5 D_{j}$

Figure 18. Radial TKE profiles for clean jet experimental and simulation.

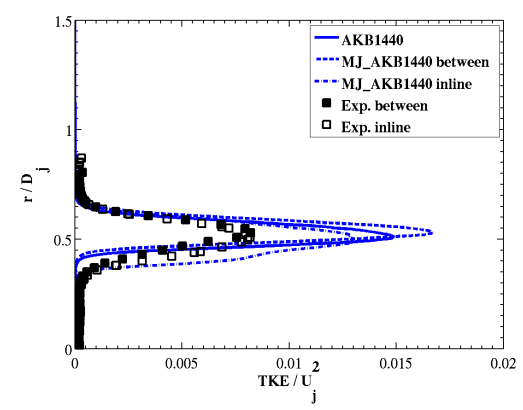

(a) $x=1 D_{j}$

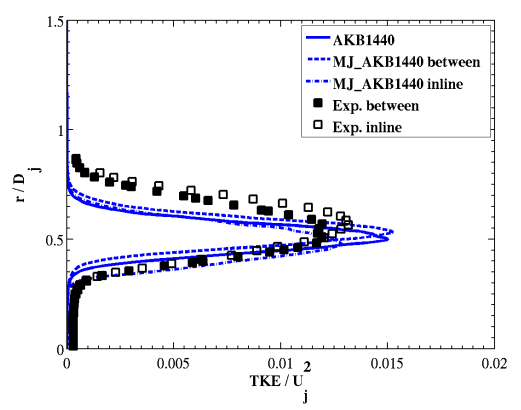

(b) $x=2 D_{j}$

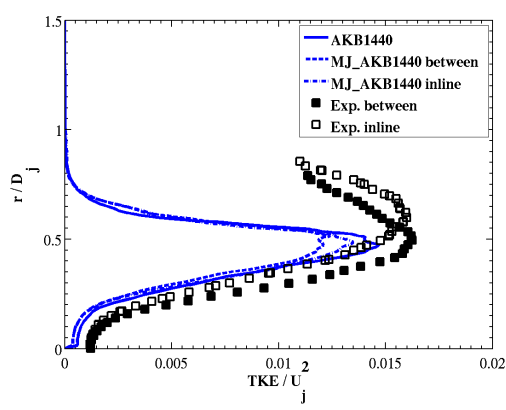

(c) $x=5 D_{j}$

Figure 19. Radial TKE profiles for microjet experimental and simulation.

MJ_AKB1440 profiles, indicating that the effect of the microjets on the turbulent structures is diminished by this axial location.

Overall the finest grid MJ_AKB1440 simulation show good agreement with experiment for mean velocity and turbulence quantities and there is little difference between these and the coarser MJ_AKB720 simulation. The following section uses the time dependent solution to compute the far-field noise.

\section{B. Aeroacoustic results}

Aeroacoustic results are presented for AKB1440 and MJ_AKB1440 simulations only. The non-uniform mesh employed in the AKB720 and MJ_AKB720 simulations clearly affects the initial shear layer development, causing non-physical variations in the azimuthal direction and will not be considered here.

Dilatation provides a qualitative view of the sound field emanating from the jet. Figure 20 shows a centreline slice of dilatation overlayed with instantaneos iso-surfaces of Mach number for the AKB1440 and MJ_AKB1440 simulations. Figure 20(a) shows fine scale waves originating near the nozzle exit and emanating in all directions with a slight downstream bias. Further downstream, much larger wavelengths can be seen that appear to propagate in the downstream direction. With the application of the microjets, Figure 20(b) shows an increase in the intensity, and a reduction in wavelength, of the waves originating from the near nozzle region. This is likely due to the presence of the microjets.

Visualisation of dilatation and related variables allows for limited quantitative analysis of the data. However, through the Ffowcs Williams-Hawking method described previously, the acoustic pressure at prescribed observer locations was calculated. Spectra from AKB1440 and MJ_AKB1440 at $20^{\circ}$ and $90^{\circ}$ are shown in Figures 21 and 22, respectively.

At $20^{\circ}$ the addition of microjets shows a small reduction in the low frequency range. Around a Strouhal number of $S t=9$ a peak in the microjet spectrum exists that is absent from the clean spectrum. A second, lower amplitude peak again appears near $S t=18$. These peaks may be attributeable to a vortex shedding mechanism of the microjets. If the microjet is considered to be a cylinder in a cross flow with a local vortex shedding frequency of $S t=0.2$, then the global frequency of this mechanism would be approximately 


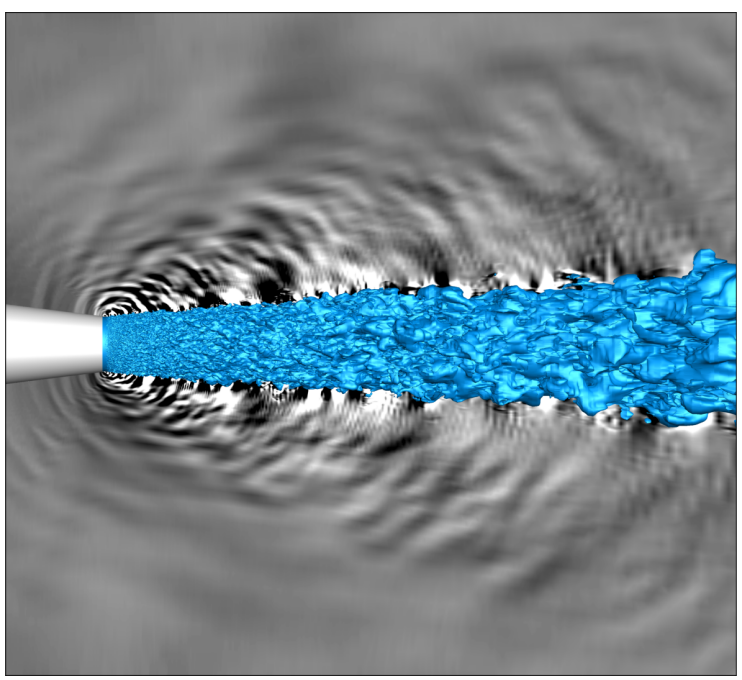

(a) AKB1440

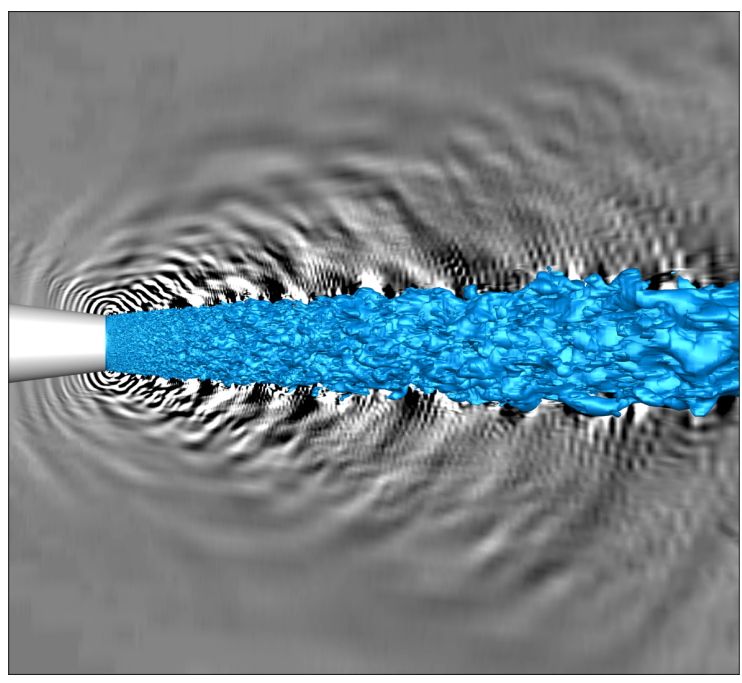

(b) MJ_AKB1440

Figure 20. Dilatation showing propagating sound waves.

$S t \approx 18$. Another consideration is that peak frequency for a jet has been shown to be around $S t=0.2$. It is possible that peaks in the spectra in Figures 21 (b) and 22 (b) are caused by the additional jet noise generated by microjets themselves.

At $90^{\circ}$ to the jet axis a more significant reduction in the low frequency range is found with the application of the microjets. Again the peak near $S t=9$ is seen in the microjet spectrum. Beyond this, the high frequencies appear to be reduced at $90^{\circ}$ from the jet axis with the microjets active.

The overall sound pressure levels for an observer located $100 D_{j}$ from the nozzle exit are plotted against downstream observer angle for the experiment and the AKB1440 and MJ_AKB1440 simulations in Figure 23. Overall, the predicted levels are overestimated by approximately 6-8 dB. Comparing the AKB1440 to the experimental OAPSL shows the general trend of the experimental noise is well captured by the simulation. Estimated overall sound pressure levels obtained from ESDU Data Item $89041^{21}$ are provided in the figure as a reference. Using the expected jet properties, the estimated OASPL values lie between the experimental and simulation results. The experimental results show a $1 \mathrm{~dB}$ reduction at $20^{\circ}$ from the jet axis, decreasing to about $0.5 \mathrm{~dB}$ at a sideline observer location. Conversely, the simulations predict a maximum noise reduction around an observer location $60^{\circ}$ from the downstream axis and almost no benefit at $20^{\circ}$. Despite the discrepancy between the experimental and simulated OASPL values, the general trends in far-field noise and effects of the microjets are well captured.

\section{Conclusions}

This work has compared the results of high resolution large eddy simulations to experimental data for a Mach 0.9 jet at a Reynolds number of 1.3 million with microjet injection. Two computational grids were used in the simulations, with one including varying azimuthal cell size. The non-uniform mesh used in the AKB720 and MJ_AKB720 simulations was demonstrated to clearly modify the laminar to turbulent transition location, instantaneous turbulent structures and turbulent kinetic energy values near the nozzle exit. Despite this effect, little impact on radial mean velocity profiles were shown. Radial velocity profiles located at $x=1,2$, and $5 D_{j}$ agreed well with the experimental profiles at the same locations for both clean and microjet cases. Radial turbulent kinetic energy profiles agreed well with the experiment, capturing the effect of the microjet injection on the shape of the profile and location of the peak TKE. The introduction of the microjets clearly affected both the spectral SPL and overall sound pressure levels. With the microjets active, a peak in the spectra occurred near $S t=9$ for both sideline and downstream observer locations that was absent from the clean jet spectra. This effect of the microjets on the spectra was not observed in the experimental work. It is suspected that these peaks in the acoustic spectra may be caused by either a vortex shedding mechanism of the microjets, or the jet noise associated with the microjets. Overall sound pressure 


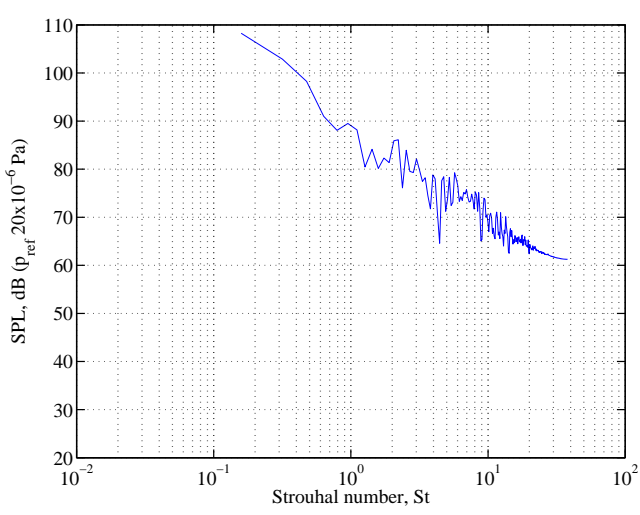

(a) AKB1440

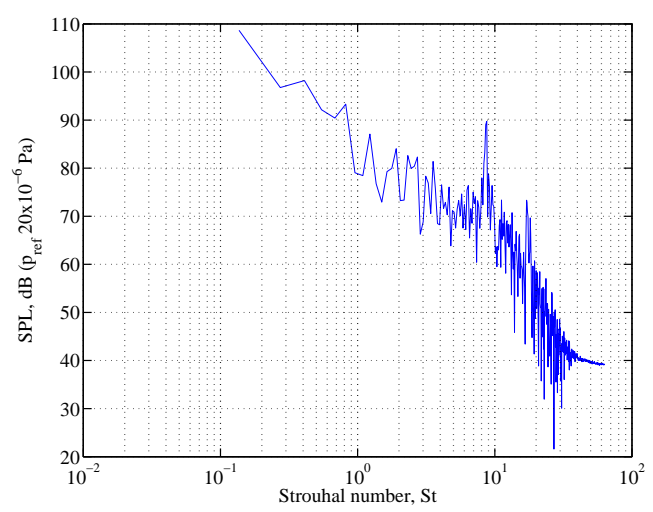

(b) MJ_AKB1440

Figure 21. Acoustic spectra at $20^{\circ}$ for AKB1440 and MJ_AKB1440.

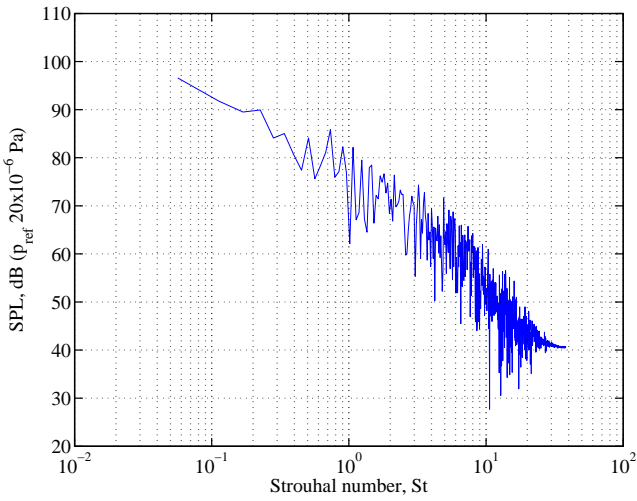

(a) AKB1440

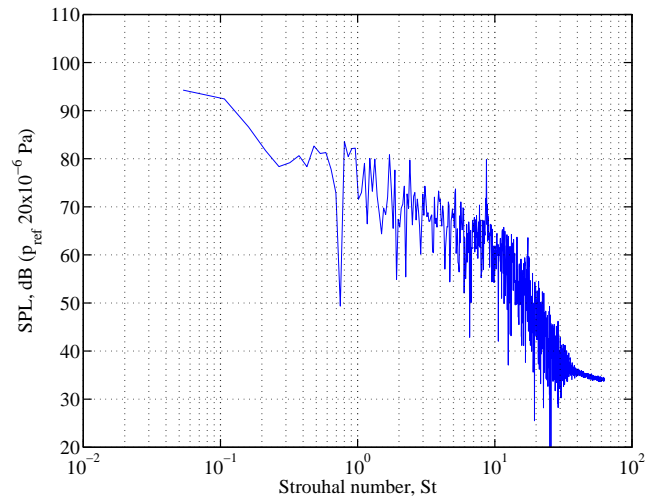

(b) MJ_AKB1440

Figure 22. Acoustic spectra at $90^{\circ}$ for AKB1440 and MJ_AKB1440.

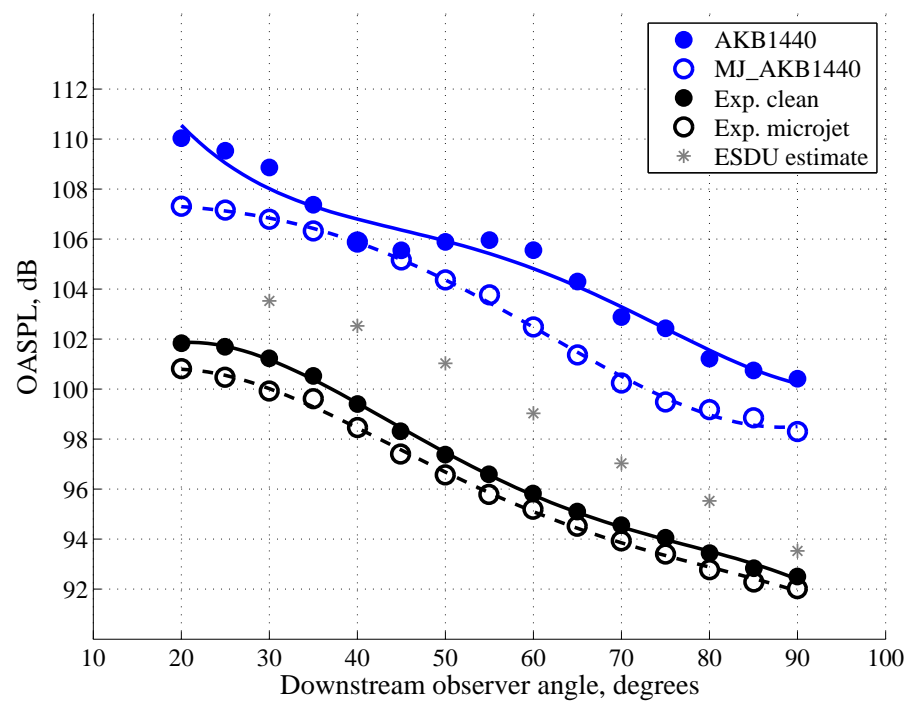

Figure 23. Overall sound pressure levels for observer at $100 D_{j}$. 
levels were generally reduced by the application of the microjets, though with larger reductions found in the more sideline directions. This result is in contrast with the experimental data, showing larger reductions in the downstream observer angles. Whilst overall sound pressure levels were overpredicted by $6-8 \mathrm{~dB}$ by the simulation, the correct behaviour with observer angle was captured and more importantly the microjets showed a reduction in OASPL of $2 \mathrm{~dB}$ across a range of angles, a similar result to that of the experiment.

\section{Acknowledgments}

The authors would like to thank Mehmet Alkislar at Boeing for providing access to the experimental data. Computing time for these calculations was provided by the UK Turbulence Consortium under funding from the EPSRC grant EP/G069581/1.

\section{References}

${ }^{1}$ Alkislar, M., "Aeroacoustics of a Mach 0.9 Jet with Chevron-Microjet Combination," 29th AIAA Aeroacoustics Conference, AIAA, Vancouver, May 2008.

${ }^{2}$ Alkislar, M., Krothapalli, A., and Butler, G., "The effect of streamwise vortices on the aeroacoustics of a Mach 0.9 jet," Journal of Fluid Mechanics, Vol. 578, 2007, pp. 139-169.

${ }^{3}$ Bridges, J. and Brown, C., "Parametric Testing of Chevrons on Single Flow Hot Jets," 10th Aeroacoustics Conference, AIAA, Manchester, United Kingdom, May 2004.

${ }^{4}$ Castelain, T., Sunyach, M., Juve, D., and Bera, J.-C., "Jet-Noise Reduction by Impinging Microjets: An Acoustic Investigation Testing Microjet Parameters," AIAA Journal, Vol. 46, No. 5, May 2008, pp. 1081-1087.

${ }^{5}$ Åberg, M., Szasz, R., Fuchs, L., and Gutmark, E., "Numerical Study of Fluidic Injection for Noise Reduction," 45th AIAA Aerospace Sciences Meeting and Exhibit, AIAA, Reno, Nevada, January 2007.

${ }^{6}$ Huet, M., Fayard, B., Rahier, G., and Vuillot, F., "Numerical Investigation of the Micro-Jets Efficiency for Jet Noise Reduction," 15th Aeroacoustics Conference, AIAA, Miami, Florida, May 2009.

${ }^{7}$ Lew, P., Najafiyazdi, A., and Mongeau, L., "Unsteady Numerical Simulation of a Round Jet with Impinging Microjets for Noise Suppression," 48th AIAA Aerospace Sciences Meeting, AIAA, Orlando, Florida, January 2010.

${ }^{8}$ Najafi-Yazdi, A., Lew, P.-t., and Mongeau, L., "Large Eddy Simulation of Jet Noise Suppression by Impinging Microjets," 17th AIAA/CEAS Aeroacoustics Conference, No. June, Portland, Oregon, 2011.

${ }^{9}$ Enomoto, S., Yamamoto, K., Yamashita, K., Tanaka, N., Oba, Y., and Oishi, T., "Large-Eddy Simulation of HighSubsonic Jet flwo with Microjet Injection," 17th AIAA/CEAS Aeroacoustics Conference, AIAA, Portland, Oregon, June 2011.

${ }^{10}$ Shur, M., Spalart, P., and Strelets, M., "LES-based evaluation of a microjet noise reduction concept in static and flight conditions," Procedia Engineering, Vol. 6, Jan. 2010, pp. 44-53.

${ }^{11}$ Rife, M. and Page, G., "Large Eddy Simulation of High Reynolds Number Jets with Microjet Injection," 17th AIAA/CEAS Aeroacoustics Conference, AIAA, Portland, Oregon, June 2011.

${ }^{12}$ Andersson, N., Eriksson, L.-E., and Davidson, L., "Large-Eddy Simulation of a Mach 0.75 Jet," 9th AIAA/CEAS Aeroacoustic Conference, Hilton Head, SC, 2003.

${ }^{13}$ Andersson, N., Eriksson, L.-E., and Davidson, L., "A Study of Mach 0 . 75 Jets and Their Radiated Sound Using Large-Eddy Simulation," 10th AIAA/CEAS Aeroacoustics Conference, Manchester, UK, 2004.

${ }^{14}$ Andersson, N., Eriksson, L.-E., and Davidson, L., "Investigation of an isothermal Mach 0.75 jet and its radiated sound using large-eddy simulation and Kirchhoff surface integration," International Journal of Heat and Fluid Flow, Vol. 26, No. 3, 2005, pp. 393-410.

${ }^{15}$ Andersson, N., Eriksson, L.-E., and Davidson, L., "Large-eddy simulation of subsonic turbulent jets and their radiated sound," AIAA journal, Vol. 43, No. 9, 2005, pp. 1899.

${ }^{16}$ Karabasov, S. A., Afsar, M. Z., Hynes, T. P., Dowling, A. P., McMullan, W. A., Pokora, C. D., Page, G. J., and McGuirk, J. J., "Using Large Eddy Simulation within and Acoustic Analogy Approach for Jet Noise Modelling," 14th AIAA/CEAS Aeroacoustics Conference, AIAA, Vancouver, Canada, May 2008.

${ }^{17}$ Shur, M., Spalart, P., and Strelets, M., "Noise prediction for increasingly complex jets. Part I: Methods and tests," International Journal of Aeroacoustics, 4, Vol. 3, No. 4, 2005, pp. 213-246.

${ }^{18}$ Shur, M., Spalart, P., and Strelets, M., "Noise prediction for increasingly complex jets. Part II: Applications," International Journal of Aeroacoustics, 4, Vol. 3, No. 4, 2005, pp. 247-266.

${ }^{19}$ Brentner, K. S. and Farassat, F., "Analytical Comparison of the Acoustic Analogy and Kirchoff Formulation for Moving Surfaces," AIAA Journal, Vol. 36, No. 8, 1998, pp. 1379-1386.

${ }^{20}$ Morgans, A. S., Transonic Helicopter Noise, Ph.D. thesis, University of Cambridge, 2003.

${ }^{21} \mathrm{ESDU}$, "Estimation of subsonic far-field jet-mixing noise from single-stream circular nozzles," Tech. Rep. December 1989, ESDU, 1990. 